\begin{tabular}{|l|l|l|l|l|l|}
\hline MUNIBE Antropologia-Arkeologia & $n^{\circ} 67$ & $295-312$ & DONOSTIA & 2016 & ISSN 1132-2217 • eISSN 2172-4555 \\
\hline
\end{tabular}

\title{
Epipaleolítico y Mesolítico en la vertiente sur de los Pirineos desde la perspectiva de la industria lítica
}

\author{
Epipaleolithic and Mesolithic on the southern slopes \\ of the Pyrenees from the lithic industry perspective
}

\author{
PALABRAS CLAVES: Aziliense, Epimagdaleniense, Sauveterroide, Mesolítico de muescas y denticulados; Mesolítico geométrico, Cataluña, valle \\ del Ebro. \\ GAKO-HITZAK: Aziliar, Epimagdaleniar, Sauveterroide, Hozka eta dentikulatu Mesolitoa, Mesolito geometrikoa, Katalunia, Ebro arroa \\ KEY WORDS: Azilian, Epimagdalenian, Sauveterroid, Notches and Denticulate Mesolithic, Geometric Mesolithic, Catalonia, Ebro basin.
}

\section{Adriana SOTO(1), Alfonso ALDAY(2), Xavier MANGADO(3) y Lourdes MONTES ${ }^{(4)}$}

\section{RESUMEN}

Se analiza la evolución industrial del Epipaleolítico y Mesolítico de la vertiente sur de los Pirineos, ofreciéndose el estado actual de conocimiento sobre las distintas tradiciones industriales que se suceden: Epimagdaleniense y Aziliense; Sauveterroide; Mesolítico de muescas y denticulados y Mesolítico geométrico. Ello permite definir los distintos intereses productivos y las diferentes estrategias desarrolladas en cada fase, destacándose, a pesar de lógicos particularismos regionales, la notable homogeneidad cultural del territorio analizado. Finalmente, se exponen los principales interrogantes existentes.

\section{LABURPENA}

Pirinioen hegoaldeko isurialdean Epipaleolitoan eta Mesolitoan izandako industria-bilakaera aztertu dugu eta han izandako tradizio industrialei buruz gaur egun dakiguna eskaintzen dugu. Hauek dira hiru tradizio horiek: Epimagdaleniarra eta Aziliarra; Sauveterroidea; Mesolitikoa hozka eta dentikulatuekin eta Mesolitiko geometrikoa. Horrek aukera ematen du aldi bakoitzean izandako ekoizpen-interesak eta garatutako estrategiak zehazteko eta eremu bakoitzari berezitasunak badiren arren, aipagarria da aztertutako lurraldeko homogeneotasun kulturala. Azkenik, gaiari lotuta argitzeke dauden zalantza nagusiak azaldu ditugu.

\section{ABSTRACT}

In this paper we analyze the evolution of lithic industries in the Epipaleolithic and Mesolithic period in southern slopes of the Pyrenees, offering a current state of knowledge about the different industrial traditions developed: the Epimagdalenian and Azilian; Sauveterroid; Notches and denticulate Mesolithic and Geometric Mesolithic. The first technological tradition is characterized by blade production and the predominance of backed points and end-scrapers. In this phase raw material's supply take place in a more restricted territory, continuing with the regionalization process started at the end of Upper Magdalenian. Also, it is noticeable the continuity of simplification and microlithisation tendencies in production dynamics. The Sauveterroid ensembles continue with similar strategies, but new types of projectiles appear: geometrics. By contrast, the Notches and denticulate Mesolithic implies a profound change in lithic system organization. Blades are replaced by flake productions, and lithic projectiles disappear. Retouched industry is now dominated by notches and denticulated pieces. Exploitation and management of lithic resources changes completely, developing new strategies like recycling. Finally, the development of Geometric Mesolithic means the recovery of regular and standardized blade production and the manufacture of stone projectiles, dominated by geometrics. This review allows us to define the different strategies developed in the production of lithic industry at every stage, remarking, although logical regional particularities, the cultural homogeneity of the analyzed territory.

\section{1.- INTRODUCCIÓN}

La investigación desarrollada en los últimos años en el noreste peninsular ha permitido un significativo avance en el conocimiento de las sociedades de finales del Pleistoceno y de los inicios del Holoceno. Las actuaciones de distintos equipos han incrementado considera- blemente los datos, llenando de contenido este periodo. Los trabajos han centrado su actividad en la Cataluña prepirenaica, valles somontanos, Bajo Aragón, oeste de Navarra y Álava, áreas donde se concentran buena parte de los yacimientos. A pesar del carácter principalmente regional de los estudios, se han identificado tendencias

\footnotetext{
(1) E-mail: asoto.sebastian@yahoo.es.

(2) Dpto. de Geografía, Prehistoria y Arqueología. Universidad del País Vasco - Euskal Herriko Unibertsitatea (UPV-EHU).

(3) Departament de Prehistòria, Història Antiga i Arqueologia, Universitat de Barcelona.

(4) Dpto. Ciencias de la Antigüedad, Área de Prehistoria, Universidad de Zaragoza.
} 
culturales comunes, evidencia de una notable interacción entre las comunidades que habitaban la vertiente surpirenaica, tanto en zonas de montaña como en las tierras bajas, que aparecen como sociedades complementarias.

En este sentido, el artículo pretende superar las ópticas habituales, más regionales, sumando y sintetizando a la par toda la cuenca del Ebro y el resto del territorio catalán. Un esfuerzo todavía no realizado.

Desde las bases establecidas por Fortea (1973), se propone hoy una secuencia evolutiva de las estrategias productivas y de gestión del utillaje lítico, diferenciando cuatro tradiciones: a) Epipaleolítico microlaminar o Epimagdaleniense (EM) y Aziliense (AZI); b) Sauveterroide (SAU); c) Mesolítico de muescas y denticulados (MMD); y d) Mesolítico geométrico (MG). Los interrogantes que plantean estos cambios tecnológicos atañen desde el modo en el que se organizaban los sistemas líticos, sus características, su evolución o funcionalidad, hasta su articulación con otros cambios en la organización de aquellas sociedades.

El artículo plantea y quiere resolver las contingencias de las industrias líticas, marcadas por la variabilidad de estrategias adoptadas entre finales del Allerød a la llegada del Neolítico, al sur de la cadena pirenaica, detallando los caracteres más significativos, evaluando los momentos de transición y observando las diferencias y semejanzas internas. El discurso final, aunque técnico, al reflejar la evolución industrial a lo largo de este periodo, pretende servir de soporte a otros estudios, globales o regionales, de carácter más explicativo, pero necesitados de un armazón que estructure las hipótesis planteadas.

\section{2.- PRESENTACIÓN DE DATOS}

Contamos con una gran cantidad de yacimientos y niveles -respectivamente más de 50 y 200 -, en la mayoría de los casos con dataciones adecuadas que permiten situar el desarrollo temporal de cada una de las tradiciones (Tabla 1 y Fig.1). No obstante, el grueso de la información proviene de un tipo particular de yacimiento, los abrigos bajo roca. El énfasis en la investigación sobre estos lugares deriva de su relativa fácil localización y de su oferta de estratigrafías de largo recorrido con materiales en aceptable conservación. Este enfoque nos devuelve una imagen distorsionada de la realidad, por cuanto que en las áreas sin abrigos pareciera que hubiera una falta de población y porque
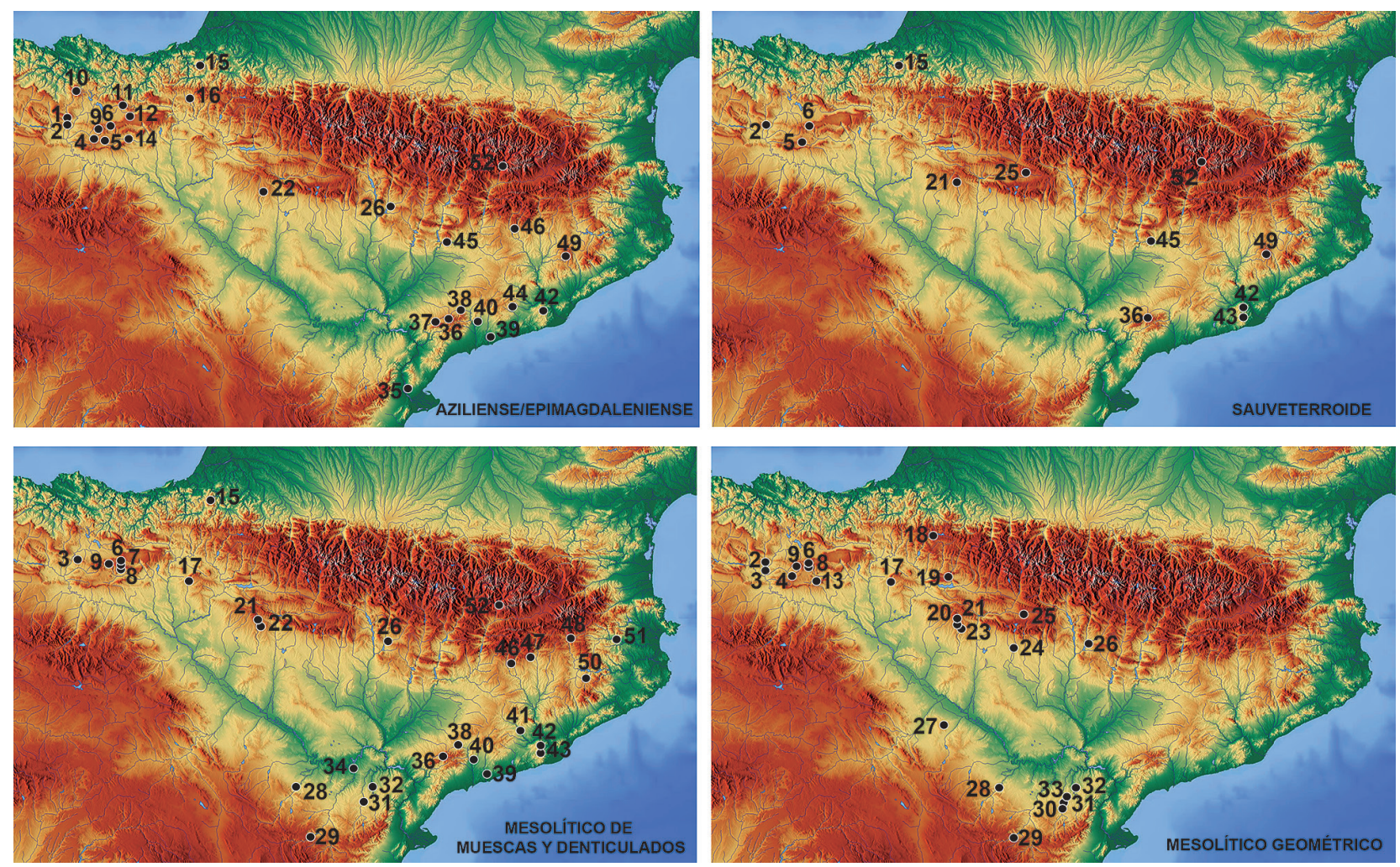

Fig. 1. Evolución del poblamiento según las fases industriales. Evolution of settlement depending on industrial phases 1. Berniollo; 2. Socuevas; 3. Fuente Hoz; 4. Montico de Charratu; 5. Martinarri; 6. Atxoste; 7. Kanpanoste; 8. Kanpanoste Goikoa; 9. Mendandia; 10. Urratxa III; 11. Anton Koba; 12. Portugain; 13. La Peña; 14. Las Orcillas; 15. Berroberria; 16. Abauntz; 17. Artusia; 18. Aizpea; 19. Padre Areso; 20. Valcervera; 21. Peña-14; 22. Legunova; 23. Rambla de Legunova; 24. Espantalobos; 25. El Esplugón; 26. Forcas I y II; 27. Cabezo de la Cruz; 28. Los Baños; 29. Ángel 1 y 2; 30. Botiquería dels Moros; 31. Pontet; 32. Costalena; 33. Els Secans; 34. Plano del Pulido; 35. Cova del Vidre; 36. Filador; 37. Els Colls; 38. Molí del Salt; 39. La Cativera; 40. Picamoixons; 41. Abric Agut; 42. Can Sadurní; 43. Marge del Moro; 44. Guineu; 45. Parco; 46. Balma Guilanyà; 47. Font del Ros; 48. Sota Palou; 49. Balma del Gai; 50. Roc del Migdia; 51. Balma del Serrat del Pont; 52. Balma Margineda. 


\begin{tabular}{|c|c|c|c|c|c|c|c|c|c|}
\hline Yacimiento & Nivel & $\mathrm{AC}$ & Datación & Desv & Ref.lab. & Cal BP (95\%) & $M$ & $\mathbf{P}$ & Biblio \\
\hline Berniollo & II & EM/AZI & 9940 & 490 & I-14786 & $12930-10050$ & $\mathrm{HH}$ & conv & Mariezkurrena, 1990 \\
\hline Socuevas & IV & EM/AZI & 11130 & 50 & Beta-282215 & $13120-12840$ & $\mathrm{H}$ & AMS & inéditas \\
\hline Socuevas & III & SAU & 10550 & 50 & Beta-282214 & $12680-12360$ & $\mathrm{H}$ & AMS & inéditas \\
\hline Socuevas & III & SAU & 9260 & 50 & Beta-282213 & $10600-10240$ & $\mathrm{H}$ & AMS & inéditas \\
\hline Socuevas & II & MG & 7590 & 45 & GrA-46015 & $8750-8390$ & $\mathrm{HH}$ & AMS & inéditas \\
\hline Fuente $\mathrm{Hoz}$ & IV & MMD & 8120 & 240 & I-12895 & $9640-8400$ & $C$ & conv & Baldeón et al ., 1983a \\
\hline Fuente $\mathrm{Hoz}$ & III & MG & 7880 & 120 & I-13496 & $9110-8390$ & C & conv & Baldeón et al., 1983a \\
\hline Fuente $\mathrm{Hoz}$ & III & MG & 7140 & 120 & I-12778 & $8230-7710$ & C & conv & Baldeón et al., 1983a \\
\hline Fuente Hoz & III & MG & 7840 & 130 & I-12083 & $9090-8330$ & $\mathrm{C}$ & conv & Baldeón et al., 1983a \\
\hline Montico de Charratu & IV & EM/AZI & & & & & & & Baldeón et al., 1983a \\
\hline Montico de Charratu & III & EM/AZI & & & & & & & Baldeón et al., 1983a \\
\hline Montico de Charratu & II & MG & & & & & & & Baldeón et al., 1983a \\
\hline Martinarri & 102 & SAU & 8455 & 45 & GrA-46014 & $9560-9400$ & $\mathrm{H}$ & AMS & Alday et al., 2012 \\
\hline Martinarri & 101 & SAU & & & & & & & Alday et al ., 2012 \\
\hline Atxoste & VIb2 & EM/AZI & & & & & & & Alday, 2014 \\
\hline Atxoste & E2 & EM/AZI & 9510 & 150 & GrN-26664 & $11290-10370$ & $\mathrm{H}$ & conv & Alday, 2014 \\
\hline Atxoste & E2 & EM/AZI & 9820 & 150 & GrN-26665 & $11840-10680$ & $\mathrm{H}$ & conv & Alday, 2014 \\
\hline Atxoste & VIb & SAU & 9550 & 60 & GrA-15858 & $11190-10630$ & $\mathrm{H}$ & AMS & Alday, 2014 \\
\hline Atxoste & VIb & SAU & 9510 & 50 & GrA-35142 & $11190-10550$ & $\mathrm{H}$ & AMS & Alday, 2014 \\
\hline Atxoste & $\mathrm{E}$ & SAU & 9450 & 50 & GrA-35141 & $10850-10530$ & $\mathrm{H}$ & AMS & Alday, 2014 \\
\hline Atxoste & $\mathrm{E}$ & SAU & 9650 & 150 & GrN-26663 & $11400-10560$ & $\mathrm{H}$ & AMS & Alday, 2014 \\
\hline Atxoste & VI & MMD & 8760 & 50 & GrA-15699 & $9980-9540$ & $\mathrm{H}$ & AMS & Alday, 2014 \\
\hline Atxoste & VI & MMD & 8510 & 80 & GrA-15700 & $9600-9400$ & $\mathrm{H}$ & AMS & Alday, 2014 \\
\hline Atxoste & V & MMD & 8030 & 50 & GrA-13448 & $9090-8690$ & $\mathrm{H}$ & AMS & Alday, 2014 \\
\hline Atxoste & V & MMD & 7810 & 40 & GrA-13447 & $8670-8510$ & $\mathrm{H}$ & AMS & Alday, 2014 \\
\hline Atxoste & D & MMD & 8840 & 50 & GrA-13473 & $10250-9650$ & $\mathrm{H}$ & AMS & Alday, 2014 \\
\hline Atxoste & IV & MG & 7340 & 70 & GrA-13418 & $8360-7960$ & $\mathrm{H}$ & AMS & Alday, 2014 \\
\hline Atxoste & IV & MG & 6970 & 40 & GrA-13419 & $7920-7680$ & $\mathrm{H}$ & AMS & Alday, 2014 \\
\hline Atxoste & IIIb2 & MG & 6940 & 40 & GrA-13415 & $7870-7670$ & $\mathrm{H}$ & AMS & Alday, 2014 \\
\hline Atxoste & IIIb2 & MG & 7140 & 50 & GrA-13468 & $8040-7880$ & $\mathrm{H}$ & AMS & Alday, 2014 \\
\hline Kanpanoste & Lanhs & MMD & 7620 & 70 & GrN-22440 & $8580-8300$ & $\mathrm{H}$ & conv & Cava, 2004a \\
\hline Kanpanoste & Lanhi & MMD & 7920 & 100 & GrN-22442 & $9080-8480$ & $\mathrm{H}$ & conv & Cava, 2004a \\
\hline Kanpanoste & Lanhi & MMD & 8200 & 70 & GrN-22441 & $9390-8950$ & $\mathrm{H}$ & conv & Cava, 2004a \\
\hline Kanpanoste Goikoa & Illinf & MMD & 7860 & 330 & GrN-20455 & $9540-8020$ & $\mathrm{H}$ & conv & Alday, 1998 \\
\hline Kanpanoste Goikoa & Illinf & MMD & 7620 & 80 & GrN-20215 & $8580-8300$ & $\mathrm{H}$ & conv & Alday, 1998 \\
\hline Kanpanoste Goikoa & III & MG & 6550 & 260 & GrN-20298 & $7900-6900$ & $\mathrm{H}$ & conv & Alday, 1998 \\
\hline Kanpanoste Goikoa & III & MG & 6360 & 70 & GrN-20214 & $7460-7140$ & $\mathrm{H}$ & conv & Alday, 1998 \\
\hline Mendandia & V & EM/AZI & 8500 & 60 & GrA-6874 & $9580-9420$ & $\mathrm{HH}$ & AMS & Alday, 2006a \\
\hline Mendandia & IV & MMD & 7810 & 50 & GrN-22744 & $8710-8470$ & $\mathrm{HH}$ & conv & Alday, 2006a \\
\hline Mendandia & IV & MMD & 7780 & 40 & GrN-22745 & $8650-8450$ & $\mathrm{HH}$ & conv & Alday, 2006a \\
\hline Mendandia & Illinf & MG & 7620 & 50 & GrN-22743 & $8530-8330$ & $\mathrm{HH}$ & conv & Alday, 2006a \\
\hline Urratxa III & II & $\mathrm{EM} / \mathrm{AZI}$ & 10240 & 100 & Ua-11433 & $12490-11530$ & $\mathrm{H}$ & AMS & Muñoz y Berganza, 1997 \\
\hline Anton Koba & VIII & $\mathrm{EM} / \mathrm{AZI}$ & 11800 & 330 & I-16236 & $14550-12950$ & $\mathrm{H}$ & conv & Armendáriz, 1997 \\
\hline Anton Koba & VIII & $\mathrm{EM} / \mathrm{AZI}$ & 11700 & 180 & I-17479 & $13900-13180$ & $\mathrm{H}$ & conv & Armendáriz, 1997 \\
\hline Portugain & & $\mathrm{EM} / \mathrm{AZI}$ & 10370 & 90 & GrN-14097 & $12550-11910$ & $\mathrm{HH}$ & conv & Barandiarán y Cava, 2008 \\
\hline La Peña & d & MG & 7890 & 120 & BM-2363 & $9100-8420$ & $\mathrm{H}$ & conv & Cava y Beguiristáin, 1991-1992 \\
\hline Las Orcillas & 1 & $\mathrm{EM} / \mathrm{AZI}$ & 8610 & 50 & Beta-252434 & $9690-9490$ & $\mathrm{H}$ & conv & Fernández Eraso et al., 2010 \\
\hline Berroberria & D & $\mathrm{EM} / \mathrm{AZI}$ & 10300 & 170 & GrN.19608 & $12690-11410$ & $\mathrm{H}$ ? & conv & Barandiarán, 1993-1994 \\
\hline Berroberria & $\mathrm{D}$ & $\mathrm{EM} / \mathrm{AZI}$ & 10160 & 410 & BM.2371 & $12950-10630$ & $\mathrm{HH}$ & conv & Barandiarán, 1993-1994 \\
\hline Berroberria & $\mathrm{D}$ & EM/AZI & 9740 & 190 & GrN.19607 & $11770-10490$ & $\mathrm{H}$ ? & conv & Barandiarán, 1993-1994 \\
\hline Berroberria & C & SAU & 8860 & 100 & GrN.18425 & $10300-9580$ & $\mathrm{H}$ & conv & Barandiarán, 1993-1994 \\
\hline Berroberria & C & SAU & 8630 & 70 & GrN.18426 & $9790-9470$ & $\mathrm{H}$ & conv & Barandiarán, 1993-1994 \\
\hline Berroberria & C & SAU & 8510 & 90 & GrN.16618 & $9640-9360$ & $\mathrm{HH}$ & conv & Barandiarán, 1993-1994 \\
\hline Berroberria & B & MMD & 8800 & 80 & GrN-18424 & $10240-9520$ & $\mathrm{HH}$ & conv & Barandiarán, 1993-1994 \\
\hline Berroberria & B & MMD & 8580 & 80 & GrN-18422 & $9750-9430$ & $\mathrm{HH}$ & conv & Barandiarán, 1993-1994 \\
\hline Berroberria & B & MMD & 8580 & 80 & GrN-18423 & $9750-9430$ & $\mathrm{HH}$ & conv & Barandiarán, 1993-1994 \\
\hline Berroberria & B & MMD & 8470 & 80 & GrN-16619 & $9600-9320$ & $\mathrm{HH}$ & conv & Barandiarán, 1993-1994 \\
\hline Abauntz & $d$ & EM/AZI & 9530 & 300 & Ly-1964 & $11670-10030$ & $\mathrm{HH}$ & conv & Utrilla, 1982-1983 \\
\hline Aizpea & 1 & MG & 7790 & 70 & GrN-16620 & $8750-8390$ & $\mathrm{HH}$ & conv & Barandiarán y Cava, 2001 \\
\hline Aizpea & 1 & MG & 7160 & 70 & GrN-16621 & $8130-7850$ & $\mathrm{HH}$ & conv & Barandiarán y Cava, 2001 \\
\hline Aizpea & II & MG & 6600 & 50 & GrSA-779 & $7600-7400$ & $\mathrm{HH}$ & conv & Barandiarán y Cava, 2001 \\
\hline Aizpea & II & MG & 6830 & 70 & GrN-16622 & $7820-7540$ & $\mathrm{HH}$ & conv & Barandiarán y Cava, 2001 \\
\hline Padre Areso & V & MG & & & & & & & García Gazólaz, 2001 \\
\hline Padre Areso & IV & MG & & & & & & & García Gazólaz, 2001 \\
\hline Artusia & 1 & MMD & 8260 & 40 & Beta-374433 & $9430-9070$ & $\mathrm{Cr}$ & & García Martínez de Lagrán, et al., 2016 \\
\hline Artusia & II & MMD & 7790 & 40 & Beta-374432 & $8660-8460$ & $\mathrm{C}$ & & García Martínez de Lagrán, et al ., 2016 \\
\hline Artusia & III & MG & 7680 & 40 & Beta-374431 & $8570-8370$ & $\mathrm{Cr}$ & & García Martínez de Lagrán, et al., 2016 \\
\hline Artusia & IV & MG & & & & & & & García Martínez de Lagrán, et al., 2016 \\
\hline Artusia & v & MG & 7210 & 30 & Beta-379670 & $8070-7950$ & $\mathrm{C}$ & & García Martínez de Lagrán, et al., 2016 \\
\hline
\end{tabular}




\begin{tabular}{|c|c|c|c|c|c|c|c|c|c|}
\hline Yacimiento & Nivel & $\mathrm{AC}$ & Datación & Desv & Ref.lab. & Cal BP (95\%) & $\mathbf{M}$ & $\mathbf{P}$ & Biblio \\
\hline Valcervera & $b$ & MG & 7035 & 45 & GrA-45763 & $7970-7770$ & $\mathrm{H}$ & AMS & Domingo y Montes, 2009 \\
\hline Valcervera & $b$ & MG & 6995 & 40 & GrA-45783 & $7980-7700$ & $\mathrm{H}$ & AMS & Domingo y Montes, 2009 \\
\hline Valcervera & $b$ & MG & 6815 & 45 & GrA-27876 & $7730-7570$ & C & AMS & Domingo y Montes, 2009 \\
\hline Peña 14 & $d$ & SAU & 10630 & 100 & $\mathrm{GrN}-26000$ & $12780-12340$ & C & conv & Montes 2001-2002 \\
\hline Peña 14 & $d$ & SAU & 10430 & 190 & GrN-26001 & $12550-11910$ & C & conv & Montes 2001-2002 \\
\hline Peña 14 & d & SAU & 10160 & 130 & GrN-25096 & $12320-11240$ & C & conv & Montes 2001-2002 \\
\hline Peña 14 & $\mathrm{~b}$ & MMD & 8780 & 110 & GrN-25098 & $10260-9460$ & C & conv & Montes, 2001-2002 \\
\hline Peña 14 & $b$ & MMD & 8340 & 130 & GrN-25097 & $9600-9000$ & C & conv & Montes, 2001-2002 \\
\hline Peña 14 & $b$ & MMD & 8000 & 90 & GrN-25998 & $9130-8570$ & C & conv & Montes, 2001-2002 \\
\hline Peña 14 & $b$ & MMD & 8000 & 80 & GrN-25999 & $9110-8590$ & C & conv & Montes, 2001-2002 \\
\hline Peña 14 & $\mathrm{a}$ & MG & 7660 & 90 & GrN-25094 & $8630-8310$ & C & conv & Montes, 2001-2002 \\
\hline Legunova & $\mathrm{m}$ & EM/AZI & 10760 & 60 & GrA-24293 & $12760-12600$ & C & AMS & Domingo y Montes, 2009 \\
\hline Legunova & 1 & MMD & 8200 & 50 & GrA-24292 & $9340-8980$ & C & AMS & Montes, 2004 \\
\hline Legunova & 1 & MMD & 8250 & 60 & GrA-22086 & $9460-9020$ & C & AMS & Montes, 2004 \\
\hline Legunova & 2 & MMD & 8800 & 40 & GrA-24294 & $10010-9650$ & C & AMS & Montes, 2004 \\
\hline Rambla de Legunova & 2 & MG & 7235 & 45 & GrA-47886 & $8200-7920$ & C & AMS & Montes et al., 2015 \\
\hline Rambla de Legunova & 2 & MG & 7260 & 45 & GrA-61768 & $8200-7960$ & $\mathrm{H}$ & AMS & Montes et al ., 2015 \\
\hline Espantalobos & e & MG & 7900 & 50 & Beta -361625 & $9030-8510$ & C & AMS & Montes et al ., 2016 \\
\hline Espantalobos & c & MG & 7390 & 40 & Beta-361624 & $8360-8120$ & $\mathrm{C}$ & AMS & Montes et al ., 2016 \\
\hline El Esplugón & 6 & SAU? & 8380 & 40 & Beta 306722 & $9520-9280$ & $\mathrm{H}$ & AMS & Utrilla et al. , 2012 \\
\hline El Esplugón & 5 & SAU? & 8015 & 45 & GrA-59633 & $9060-8700$ & C & AMS & Utrilla et al ., 2016 \\
\hline El Esplugón & 5 & SAU? & 7860 & 40 & Beta -306725 & $8780-8540$ & $\mathrm{H}$ & AMS & Utrilla et al., 2012 \\
\hline El Esplugón & 4 & MG & 7620 & 40 & GrA-59632 & $8480-8360$ & C & AMS & Utrilla et al., 2016 \\
\hline El Esplugón & 3bajo & MG & 6950 & 50 & Beta -306723 & $7900-7660$ & $\mathrm{H}$ & AMS & Utrilla et al. , 2012 \\
\hline El Esplugón & 3 sup $/ 3$ & $\mathrm{MG} / \mathrm{N}$ & 6730 & 40 & Beta -313517 & $7670-7510$ & $\mathrm{H}$ & AMS & Utrilla et al. , 2012 \\
\hline Forcas I & 10 & EM/AZI & 11015 & 45 & GrA-32955 & $13040-12720$ & $\mathrm{H}$ & AMS & Utrilla y Mazo, 2014 \\
\hline Forcas I & 9 & EM/AZI & 9715 & 75 & GrN-17785 & $11340-10740$ & C & conv & Utrilla y Mazo, 2014 \\
\hline Forcas I & 7 & EM/AZI & 9360 & 140 & GrN-17784 & $11080-10160$ & $\mathrm{C}$ & conv & Utrilla y Mazo, 2014 \\
\hline Forcas II & $\mathrm{lb}$ & MMD & 8650 & 70 & CAMS-5354 & $9830-9470$ & C & AMS & Utrilla y Mazo, 2014 \\
\hline Forcas II & II & MG & 7150 & 40 & Beta -250944 & $8030-7910$ & $\mathrm{H}$ & conv & Utrilla y Mazo, 2014 \\
\hline Forcas II & II & MG & 7240 & 40 & GrN-22686 & $8190-7950$ & C & conv & Utrilla y Mazo, 2014 \\
\hline Forcas II & IV & MG & 7000 & 40 & Beta -290932 & $7970-7730$ & $\mathrm{H}$ & conv & Utrilla y Mazo, 2014 \\
\hline Forcas II & IV & MG & 7090 & 340 & Beta-59995 & $8590-7310$ & C & conv & Utrilla y Mazo, 2014 \\
\hline Cabezo de la Cruz & UE1351 & MG & 7130 & 130 & GrN-29134 & $8240-7680$ & C & conv & Rodanés y Picazo, 2013 \\
\hline Cabezo de la Cruz L & UE1397 & MG & 7150 & 70 & GrN-29135 & $8110-7830$ & $\mathrm{C}$ & conv & Rodanés y Picazo, 2013 \\
\hline Los Baños & $1 b$ & MMD & & & & & & & Utrilla y Rodanés, 2004 \\
\hline Los Baños & 2b1 & MMD & 8040 & 50 & GrA-21556 & $9100-8700$ & C & AMS & Utrilla y Rodanés, 2004 \\
\hline Los Baños & $2 b 1$ & MMD & 7840 & 100 & GrN-24299 & $8350-7790$ & C & conv & Utrilla y Rodanés, 2004 \\
\hline Los Baños & $2 b 1$ & MG & 7740 & 50 & GrA-21552 & $8630-8390$ & C & AMS & Utrilla y Rodanés, 2004 \\
\hline Los Baños & $2 \mathrm{~b} 3$ & MG & 7570 & 100 & GrN-24300 & $8580-8140$ & C & conv & Utrilla y Rodanés, 2004 \\
\hline Los Baños & 2b3inf & MG & 7550 & 50 & GrA-21551 & $8440-8280$ & C & AMS & Utrilla y Rodanés, 2004 \\
\hline Los Baños & 2 b3sup & MG & 7350 & 50 & GrA-21550 & $8350-7990$ & C & AMS & Utrilla y Rodanés, 2004 \\
\hline Ángel 1 & $8 d$ & MMD & 8390 & 60 & GrA-22826 & $9540-9260$ & C & AMS & Utrilla et al., 2009 \\
\hline Ángel 1 & $8 d$ & MMD & 8210 & 210 & GrN-15519 & $9670-8550$ & C & conv & Utrilla et al., 2009 \\
\hline Ángel 1 & $8 d$ & MMD & 8150 & 170 & GrN-15520 & $9560-8560$ & C & conv & Utrilla et al., 2009 \\
\hline Ángel 1 & $8 d$ & MMD & 8060 & 270 & GrN-15518 & $9630-8310$ & C & conv & Utrilla et al., 2009 \\
\hline Ángel 1 & 8binf & MG & & & & & & & Utrilla et al., 2009 \\
\hline Ángel 1 & $8 c$ & MG & 7435 & 45 & GrA-27274 & $8390-8150$ & $\mathrm{C}$ & AMS & Utrilla et al., 2009 \\
\hline Ángel 1 & $8 c$ & MG & 7955 & 45 & GrA-27278 & $9060-8580$ & C & AMS & Utrilla et al., 2009 \\
\hline Ángel 2 & $2 b$ & MMD & 8310 & 60 & GrA-22836 & $9510-9110$ & C & AMS & Utrilla et al., 2009 \\
\hline Ángel 2 & $2 \mathrm{a} 2$ & MG? & 6390 & 40 & Beta -254048 & $7460-7220$ & $\mathrm{H}$ & conv & Utrilla et al ., 2009 \\
\hline Botiq. dels Moros & 2 & MG & 7600 & 50 & GrA-13265 & $8490-8330$ & $\mathrm{H}$ & AMS & Barandiarán y Cava, 2000 \\
\hline Botiq. dels Moros & 2 & MG & 7550 & 200 & Ly-1198 & $8780-7940$ & $\mathrm{CC}$ & conv & Barandiarán y Cava, 2000 \\
\hline Botiq. dels Moros & 4 & MG & 6830 & 50 & GrA-13267 & $7770-7570$ & $\mathrm{H}$ & AMS & Barandiarán y Cava, 2000 \\
\hline Pontet & $\mathrm{i}$ & MMD & & & & & & & Mazo y Montes, 1992 \\
\hline Pontet & $\mathrm{g}$ & MMD & & & & & & & Mazo y Montes, 1992 \\
\hline Pontet & $\mathrm{e}$ & MG & 7340 & 70 & GrN-16313 & $8360-7960$ & C & conv & Mazo y Montes, 1992 \\
\hline Pontet & $\operatorname{cinf}$ & $M G / N$ & 6370 & 70 & GrN-14241 & $7470-7150$ & C & conv & Mazo y Montes, 1992 \\
\hline Costalena & $\mathrm{d}$ & MMD & & & & & & & Barandiarán y Cava, 2000 \\
\hline Costalena & c3 & $M G / N$ & 6310 & 170 & GrA-10949 & $7570-6810$ & $\mathrm{HH}$ & AMS & Barandiarán y Cava, 2000 \\
\hline Costalena & $c 3$ & $M G / N$ & 6420 & 240 & GrN-14098 & $7770-6770$ & $\mathrm{HH}$ & conv & Barandiarán y Cava, 2000 \\
\hline Els Secans & $\mathrm{IIb}$ & MG & & & & & & & Rodanés et al ., 1996 \\
\hline Els Secans & Ila & $M G / N$ & & & & & & & Rodanés et al., 1996 \\
\hline Plano del Pulido & $\mathrm{cm}$ & MMD & & & & & & & Montes et al., 2006 \\
\hline Cova del Vidre & 2 & EM/AZI & 8790 & 130 & Beta -58933 & $10290-9450$ & C & conv & Bosch, 1993 \\
\hline Cova del Vidre & 4 & MG? & 7290 & 70 & UBAR-832 & $8240-7960$ & & & Morales et al., 2013 \\
\hline Els Colls & II & EM/AZI & 10050 & 85 & AA-8646 & $11970-11210$ & $\mathrm{H}$ & AMS & Fullola et al., 1993 \\
\hline Els Colls & II & EM/AZI & 10950 & 120 & AA-8645 & $13080-12640$ & C & AMS & Fullola et al., 1994 \\
\hline
\end{tabular}




\begin{tabular}{|c|c|c|c|c|c|c|c|c|c|}
\hline Yacimiento & Nivel & $A C$ & Datación & Desv & Ref.lab. & Cal BP (95\%) & M & $\mathbf{P}$ & Biblio \\
\hline Filador & $9 / 8$ & EM/AZI & 11000 & 55 & OXA-8660 & $13030-12710$ & $\mathrm{C}$ & conv & García-Argüelles et al ., 2005 \\
\hline Filador & $9 / 8$ & EM/AZI & 10880 & 60 & OXA-8650 & $12870-12670$ & C & conv & García-Argüelles et al ., 2005 \\
\hline Filador & 7 & SAU & 9830 & 160 & UBAR-257 & $11880-10680$ & $\mathrm{C}$ & conv & García-Argüelles et al., 2005 \\
\hline Filador & 7 & SAU & 9130 & 230 & ICEN-495 & $10940-9620$ & $\mathrm{C}$ & conv & García-Argüelles et al., 2005 \\
\hline Filador & $6 / 5$ & SAU & 9988 & 97 & AA-13412 & $11890-11130$ & C & AMS & García-Argüelles et al ., 2005 \\
\hline Filador & 4 & SAU & 10020 & 80 & AA-8647 & $11880-11200$ & $\mathrm{H}$ & AMS & García-Argüelles et al ., 2005 \\
\hline Filador & 4 & SAU & 9460 & 190 & UBAR-284 & $11330-10210$ & $\mathrm{C}$ & conv & García-Argüelles et al ., 2005 \\
\hline Filador & 3 & SAU & & & & & & & García-Argüelles et al ., 2005 \\
\hline Filador & 2 & MMD & 8515 & 50 & Оха -8658 & $9560-9440$ & $\mathrm{C}$ & AMS & García-Argüelles et al ., 2005 \\
\hline Filador & 2 & MMD & 8150 & 90 & AA-13411 & $9500-8980$ & C & AMS & García-Argüelles et al ., 2005 \\
\hline Molí del Salt & Asup & EM/AZI & 10840 & 50 & Beta-179599 & $12790-12670$ & $\mathrm{C}$ & AMS & Vaquero, 2004 \\
\hline Molí del Salt & Asup & $\mathrm{EM} / \mathrm{AZI}$ & 10990 & 50 & Beta-179598 & $13020-12700$ & C & AMS & Vaquero, 2004 \\
\hline Molí del Salt & Sup & MMD & 8040 & 40 & Beta-173335 & $9100-8700$ & $\mathrm{H}$ & AMS & Vaquero, 2004 \\
\hline La Cativera & $\mathrm{C} 3 \mathrm{~b}$ & EM/AZI & 11135 & 80 & AA-23372 & $13170-12770$ & C & AMS & Morales et al., 2013 \\
\hline La Cativera & $\mathrm{C3}$ & $\mathrm{EM} / \mathrm{AZI}$ & 11230 & 100 & AA-23371 & $13310-12870$ & C & AMS & Morales et al., 2013 \\
\hline La Cativera & $\mathrm{C} 2$ & $\mathrm{EM} / \mathrm{AZI}$ & 10660 & 120 & AA-23370 & $12800-12320$ & C & AMS & Morales et al., 2013 \\
\hline La Cativera & $\mathrm{C} 1$ & $\mathrm{EM} / \mathrm{AZI}$ & 10370 & 100 & AA-23369 & $12590-11870$ & $\mathrm{C}$ & AMS & Morales et al., 2013 \\
\hline La Cativera & B & EM/AZI & 8860 & 95 & AA-23368 & $10300-9580$ & C & AMS & Morales et al., 2013 \\
\hline La Cativera & B & EM/AZI & 8230 & 40 & Beta-281623 & $9340-9060$ & C & AMS & Morales et al., 2013 \\
\hline La Cativera & A & MMD & 7979 & 60 & AA-23367 & $9070-8590$ & C & AMS & Morales et al., 2013 \\
\hline Picamoixons & IIB & EM/AZI & 11055 & 90 & AA-5810 & $13120-12720$ & C & conv & García Catalán, et al., 2009 \\
\hline Picamoixons & IIA & MMD & 9570 & 50 & Beta-214937 & $11180-10660$ & $\mathrm{H}$ & AMS & García Catalán, et al. , 2009 \\
\hline Abric Agut & $4.7 a$ & MMD & 9185 & 60 & OxA-10049 & $10540-10180$ & C & AMS & Vaquero, 2006 \\
\hline Abric Agut & $4.7 a$ & MMD & 9660 & 110 & OxA-10064 & $11320-10640$ & C & AMS & Vaquero, 2006 \\
\hline Abric Agut & $4.7 c$ & MMD & 10805 & 60 & OxA-10050 & $11970-11290$ & $\mathrm{C}$ & AMS & Vaquero, 2006 \\
\hline Abric Agut & $4.7 c$ & MMD & 9895 & 60 & OxA-10051 & $11560-11160$ & C & AMS & Vaquero, 2006 \\
\hline Abric Agut & $4.7 c$ & MMD & 10060 & 65 & OxA-10074 & $11910-11270$ & C & AMS & Vaquero, 2006 \\
\hline Can Sadurní & 21IVe & EM/AZI & 10540 & 60 & Beta-179899 & $12680-12320$ & & AMS & Fullola et al ., 2011 \\
\hline Can Sadurní & $21 \mathrm{IVd}$ & SAU & 9360 & 40 & Beta-230734 & $10710-10470$ & & AMS & Fullola et al ., 2011 \\
\hline Can Sadurní & $19 / 20$ & MMD & 7320 & 50 & Beta-230733 & $8240-7990$ & & AMS & Fullola et al ., 2011 \\
\hline Marge del Moro & VI & SAU/MMD & 8270 & 65 & OxA-8572 & $9480-9040$ & & AMS & Fullola et al ., 2011 \\
\hline Marge del Moro & VII & SAU/MMD & 8686 & 55 & OxA-8571 & $9820-9500$ & & AMS & Fullola et al ., 2011 \\
\hline Marge del Moro & VIII & MMD & & & & & & & Fullola et al ., 2011 \\
\hline C.Guineu & III & $\mathrm{EM} / \mathrm{AZI}$ & 9850 & 80 & Gif-8439 & $11510-11110$ & $\mathrm{C}$ & conv & Fullola et al, 1993 \\
\hline Parco & Ib & EM/AZI & 11430 & 60 & OxA-8656 & $13440-12890$ & $\mathrm{CC}$ & AMS & Mangado et al., 2005 \\
\hline Parco & Ic & $\mathrm{EM} / \mathrm{AZI}$ & 11270 & 90 & OxA-8657 & $13300-12980$ & $\mathrm{CC}$ & AMS & Mangado et al ., 2005 \\
\hline Parco & la2 & SAU & 10190 & 100 & AA-14310 & $12270-11430$ & C & AMS & Mangado et al., 2005 \\
\hline Parco & la2 & SAU & 10930 & 100 & Gif-95562 & $13050-12650$ & C & AMS & Mangado et al., 2005 \\
\hline Parco & la2 & SAU & 10770 & 110 & Gif-95563 & $12840-12520$ & $\mathrm{C}$ & AMS & Mangado et al ., 2005 \\
\hline Parco & la2 & SAU & 10420 & 110 & Gif-95564 & $12670-11910$ & & AMS & Mangado et al ., 2005 \\
\hline B. de Guilanyà & K & EM/AZI & 12310 & 40 & Beta-247708 & $14500-14060$ & C & AMS & Martínez-Moreno y Mora, 2009 \\
\hline B. de Guilanyà & EJ & $E M / A Z I$ & 12180 & 50 & Beta-185066 & $14200-13920$ & C & AMS & Martínez-Moreno y Mora, 2009 \\
\hline B. de Guilanyà & $E$ & $E M / A Z I$ & 11110 & 40 & Beta-247706 & $13110-12830$ & C & AMS & Martínez-Moreno y Mora, 2009 \\
\hline B. de Guilanyà & $\mathrm{E}$ & EM/AZI & 11460 & 230 & UBAR-367 & $13750-12870$ & C & conv & Martínez-Moreno y Mora, 2009 \\
\hline B. de Guilanyà & $E$ & $\mathrm{EM} / \mathrm{AZI}$ & 10940 & 50 & Beta-210729 & $12950-12670$ & $\mathrm{Cr}$ & AMS & Martínez-Moreno y Mora, 2009 \\
\hline B. de Guilanyà & $\mathrm{E}-\mathrm{HB}$ & EM/AZI & 10195 & 255 & Ua-34298 & $12740-11020$ & $\mathrm{H}$ & AMS & Martínez-Moreno y Mora, 2009 \\
\hline B. de Guilanyà & $\mathrm{E}-\mathrm{HB}$ & EM/AZI & 11095 & 195 & Ua-34297 & $13310-12630$ & $\mathrm{H}$ & AMS & Martínez-Moreno y Mora, 2009 \\
\hline B. de Guilanyà & $\mathrm{C}$ & MMD & 8970 & 430 & UBAR-368 & $11240-9040$ & $\mathrm{C}$ & conv & Martínez-Moreno y Mora, 2009 \\
\hline B. de Guilanyà & $\mathrm{C}$ & MMD & 8680 & 50 & Beta-185046 & $9790-9510$ & $\mathrm{C}$ & AMS & Martínez-Moreno y Mora, 2009 \\
\hline B. de Guilanyà & C & MMD & 8640 & 50 & Beta-210730 & $9730-9490$ & $\mathrm{Cr}$ & AMS & Martínez-Moreno y Mora, 2009 \\
\hline B. de Guilanyà & C & MMD & 9410 & 60 & Beta-186168 & $10800-10480$ & C & AMS & Martínez-Moreno y Mora, 2009 \\
\hline B. de Guilanyà & $\mathrm{C} 1$ & MMD & 9840 & 50 & Beta-210728 & $11330-11170$ & $\mathrm{Cr}$ & AMS & Martínez-Moreno y Mora, 2009 \\
\hline Font del Ros & SG & MMD & 8400 & 180 & UBAR-397 & $9740-8940$ & $\mathrm{C}$ & conv & Martínez-Moreno et al., 2006-2007 \\
\hline Font del Ros & SG & MMD & 8270 & 200 & UBAR-329 & $9670-8710$ & C & conv & Martínez-Moreno et al., 2006-2007 \\
\hline Font del Ros & SG & MMD & 8150 & 590 & UBAR-165 & $10560-7760$ & $\mathrm{C}$ & conv & Martínez-Moreno et al ., 2006-2007 \\
\hline Font del Ros & SG & MMD & 8050 & 150 & UBAR-185 & $9400-8480$ & $\mathrm{C}$ & conv & Martínez-Moreno et al ., 2006-2007 \\
\hline Font del Ros & SGA & MMD & 8800 & 360 & UBAR-345 & $10790-9030$ & $\mathrm{C}$ & conv & Martínez-Moreno et al ., 2006-2007 \\
\hline Font del Ros & SG1 & MMD & 8690 & 60 & Beta-210732 & $9850-9490$ & $\mathrm{Cr}$ & conv & Martínez-Moreno et al ., 2006-2007 \\
\hline Font del Ros & SG6 & MMD & 7800 & 50 & Beta-210733 & $8690-8450$ & $\mathrm{Cr}$ & conv & Martínez-Moreno et al ., 2006-2007 \\
\hline Sota Palou & $10 \mathrm{~A}$ & MMD & 8540 & 180 & UGRA-69 & $10080-9080$ & $\mathrm{C}$ & conv & Martínez-Moreno et al ., 2006-2007 \\
\hline Sota Palou & $10 \mathrm{~B}$ & MMD & 9060 & 380 & UGRA-380 & $11260-9220$ & C & conv & Martínez-Moreno et al ., 2006-2007 \\
\hline B. del Gai & $\mathrm{I} .1$ & SAU & 8930 & 140 & GifA-10028 & $10390-9590$ & & conv & García-Argüelles et al ., 2009 \\
\hline B. del Gai & 1.3 & $E M / A Z I$ & 10260 & 90 & GifA-95617 & $12430-11630$ & & conv & García-Argüelles et al., 2009 \\
\hline B. del Gai & 1.5 & $\mathrm{EM} / \mathrm{AZI}$ & 12240 & 110 & GifA-95630 & $14710-13790$ & & conv & García-Argüelles et al., 2009 \\
\hline
\end{tabular}




\begin{tabular}{|c|c|c|c|c|c|c|c|c|c|}
\hline Yacimiento & Nivel & $\mathrm{AC}$ & Datación & Desv & Ref.lab. & Cal BP (95\%) & $\mathbf{M}$ & $\mathbf{P}$ & Biblio \\
\hline Roc del Migdia & II & MMD & 8800 & 240 & UBAR-272 & $10540-9380$ & C & conv & Rodríguez e YII, 1991 \\
\hline Roc del Migdia & II & MMD & 8190 & 300 & UBAR-196 & $9800-8360$ & C & conv & Rodríguez e YII, 1991 \\
\hline Roc del Migdia & II & MMD & 7950 & 370 & UBAR-198 & $9710-8030$ & C & conv & Rodríguez e YII, 1991 \\
\hline Roc del Migdia & II & MMD & 7280 & 370 & UBAR-197 & $8850-7410$ & C & conv & Rodríguez e YII, 1991 \\
\hline B. del Serrat del Pont & 4.1 & MMD & 7330 & 40 & Beta -138589 & $8240-8000$ & & conv & Alcalde y Saña, 2008 \\
\hline B. del Serrat del Pont & 4.2 & MMD & 7770 & 50 & Beta-212541 & $8650-8410$ & & conv & Alcalde y Saña, 2008 \\
\hline B. del Serrat del Pont & 4.3 & MMD & 8060 & 40 & Beta-216834 & $9130-8730$ & & conv & Alcalde y Saña, 2008 \\
\hline B. del Serrat del Pont & 4.4 & MMD & 8130 & 40 & Beta-212542 & $9170-8970$ & & conv & Alcalde y Saña, 2008 \\
\hline B. del Serrat del Pont & 4.5 & MMD & 8310 & 40 & Beta -183017 & $9470-9190$ & & conv & Alcalde y Saña, 2008 \\
\hline B. Margineda & 10 & EM/AZI & 11870 & 110 & Ly-4898 & $13970-13450$ & C & conv & Martínez-Moreno et al ., 2006-2007 \\
\hline B. Margineda & 10 & EM/AZI & 11690 & 90 & Ly-4896 & $13730-13330$ & C & conv & Martínez-Moreno et al ., 2006-2007 \\
\hline B. Margineda & 8 sup & $\mathrm{EM} / \mathrm{AZI}$ & 11320 & 120 & Ly-4407 & $13400-12960$ & C & conv & Martínez-Moreno et al ., 2006-2007 \\
\hline B. Margineda & 8 & EM/AZI & 11230 & 170 & Ly-5418 & $13430-12710$ & C & conv & Martínez-Moreno et al ., 2006-2007 \\
\hline B. Margineda & 7 & EM/AZI & 10340 & 130 & Ly-4403 & $12640-11640$ & C & conv & Martínez-Moreno et al ., 2006-2007 \\
\hline B. Margineda & Sbase/i & SAU & 10640 & 260 & Ly-2843 & $13160-11760$ & $\mathrm{C}$ & conv & Guilaine y Martzluff, 1995 \\
\hline B. Margineda & 6 & SAU & 9250 & 160 & Ly-2842 & $10870-10070$ & C & conv & Guilaine y Martzluff, 1995 \\
\hline B. Margineda & $5 / 6$ & SAU & 8960 & 120 & Ly-4402 & $10390-9670$ & C & conv & Guilaine y Martzluff, 1995 \\
\hline B. Margineda & 4base & MMD & 8970 & 120 & Ly-4401 & $10380-9700$ & C & conv & Guilaine y Martzluff, 1995 \\
\hline B. Margineda & 4.5 & MMD & 8850 & 120 & Ly-3892 & $10320-9520$ & $\mathrm{C}$ & conv & Guilaine y Martzluff, 1995 \\
\hline B. Margineda & 4 & MMD & 8530 & 420 & Ly-2841 & $10640-8480$ & $\mathrm{C}$ & conv & Guilaine y Martzluff, 1995 \\
\hline B. Margineda & 4 & MMD & 8390 & 150 & Ly-2840 & $9670-8990$ & C & conv & Guilaine y Martzluff, 1995 \\
\hline B. Margineda & 4 sup & MMD & 8210 & 180 & Ly-3291 & $9630-8630$ & C & conv & Guilaine y Martzluff, 1995 \\
\hline
\end{tabular}

Tabla 1: Tabla con yacimientos y niveles del Aziliense, Epimagdaleniense o Epipaleolítico microlaminar, del Sauveterroide, del Mesolítico de muescas y denticulados, y del Mesolítico geométrico de la cuenca del Ebro y Cataluña. En gris clarito las referencias empleadas (desviaciones $\leq \pm 100$, salvo los casos adscritos al MG/N y los niveles 4 de Cova del Vidre y 2a2 de Ángel 2). EM-Epipaleolítico microlaminar o Epimagdaleniense; AZI-Aziliense; SAU-Sauveterroide; MMD-Mesolítico de muescas y denticulados; MG-Mesolítico geométrico. C- Carbón; CC-varios carbones; H- Hueso; HH- varios huesos; Cr- corylus / Azilian, Epimagdalenian, Sauveterroid, Notches and denticulate Mesolithic and Geometric Mesolithic archaeological sites and levels from Ebro basin and Catalonia. In grey references employed (dates with $\leq \pm 100$, MG/N levels and Cova del Vidre 4 and Ángel 22 2a2 excepted). EM-Epimagdalenian; AZI-Azilian; SAU-Sauveterroid; MMD- Notches and denticulate Mesolithic; MG- Geometric Mesolithic. C- Charcoal; CC-charcoal ensemble; H- Bone; HH- bone ensemble; Cr- corylus.

propone un modelo restringido de poblamiento y uso de los recursos. Los asentamientos al aire libre, como Berniollo, Font del Ros o Cabezo de la Cruz, aunque escasos, son ejemplos de un escenario más rico y diverso. Sin embargo, algunos de los vacíos no tienen por qué responder a defectos de la investigación, como parece ser la ausencia de la última fase mesolítica en Cataluña (Fig.1).

\section{3.- LAS INDUSTRIAS AZILIENSES Y EPIMAG- DALENIENSES/EPIPALEOLÍTICAS MICRO- LAMINARES}

\subsection{Las primeras evidencias}

Las evidencias más antiguas de conjuntos epipaleolíticos, considerando aquellos adjetivados como Epimagdalenienses, Microlaminares o Azilienses que manteniendo una estructura industrial dominada por dorsos se han alejado de los modos magdalenienses, son del noreste peninsular. Balma Guilanyà K y EJ y Balma Margineda c.10 nos sitúan en la primera mitad del Allerød (15000-14000-todas las referencias en cal BP-). Son Industrias dominadas por el binomio dorso/raspador: es significativo en los primeros su carácter apuntado y variabilidad (microgravettes, puntas curvas, dorsos triedros espesos y puntas fusiformes) y en los segundos su aspecto corto (MARTíNEZ-MORENO y MORA, 2009; MARTZLUFF, 2009; MARTZLUFF et al., 2012). Los sistemas de talla son definidos como expeditivos, con una débil incidencia de la producción laminar, y el empleo de rocas locales.
En el resto del territorio los niveles de la primera mitad del Allerød son, en su mayoría, adscritos al Magdaleniense superior final (MSF). Se ha señalado que la distinción desde la industria lítica entre el MSF y el EM/ AZI es compleja (BARANDIARÁN y CAVA 2001; CAVA, 2004b; VAQUERO, 2004). La progresividad desde un fondo común de los cambios industriales, no siempre en sincronía, dificulta el encaje de las colecciones, habiendo situaciones de transición.

En estas situaciones intermedias se reconocen tendencias que se acentuarán en la segunda mitad del Allerød y el Dryas III. En el alto y medio Ebro, hay secuencias del MSF (en Zatoya -BARANDIARÁN y CAVA, 2001-, Chaves -UTRILLA et al., 2010- o Atxoste -BARANDIARÁN et al., 2006-) con cambios en la composición y configuración de sus industrias, en las que aumentan los dorsos apuntados, curvos y espesos, o se generalizan los raspadores pequeños sobre lasca (SOTO et al., 2015). En otros casos, Anton Koba VIII, la presencia de arpones aplanados motiva su adscripción al Aziliense antiguo aunque su aparejo lítico mantiene rasgos propios de conjuntos paleolíticos (ARMENDÁRIZ, 1997). En el noreste también se aprecian situaciones que reflejan la complejidad y dilatación temporal de la azilianización/epipaleolitización. Así en Parco entre el MS/ MSF hay cambios en las estrategias de abastecimiento -regionalización- y de talla -simplificación y microlitización- (MANGADO et al., 2014), o en Hort de la Boquera II y Colls IV se han señalado esquemas de talla que se alejan de los parámetros anteriores (LANGLAIS, 2007; SOLER et al., 2009; GARCÍA-ARGÜELLES et al., 2014). 
En decir, a inicios del Allerød conviven conjuntos inscritos en el Aziliense antiguo junto a otros del MSF que han iniciado transformaciones significativas en sus estrategias de producción lítica como arranque de los rasgos que definirán las industrias epipaleolíticas. Es un proceso progresivo cuyos ritmos, mecanismos o articulación no es aún bien conocido.

\subsection{El desarrollo pleno}

Hacia el 13500/13000 todos los conjuntos se adscriben a tradiciones epipaleolíticas. La mayoría se concentran entre finales del Allerød y el Dryas III. A los momentos iniciales pertenecen Balma Guilanyà, Parco, Filador, Molí del Salt y Picamoixons en Cataluña; Legunova y
Forcas-I en Aragón; y Socuevas en el País Vasco. Para el Dryas III las referencias se reducen a Can Sadurní, La Cativera y Balma del Gai en Cataluña y Portugain y Urratxa III en el alto Ebro. En el Preboreal continúan Colls y la Guineu en el extremo oriental; y Atxoste y Forcas-I en el alto y medio Ebro. Una reflexión aparte merece la perduración de industrias microlaminares con dominio de dorsos en momentos avanzados del Boreal: con ciertas reservas en el alto Ebro, dada la pobreza del nivel V de Mendandia y de la ocupación de Las Orcillas, pero corroborado en La Cativera. Sin dataciones absolutas, o con amplias desviaciones están Montico de Charratu, Berniollo, Abauntz, Berroberria y Cova del Vidre, los cuatro primeros del sector occidental de la Cuenca, la última del oriental.

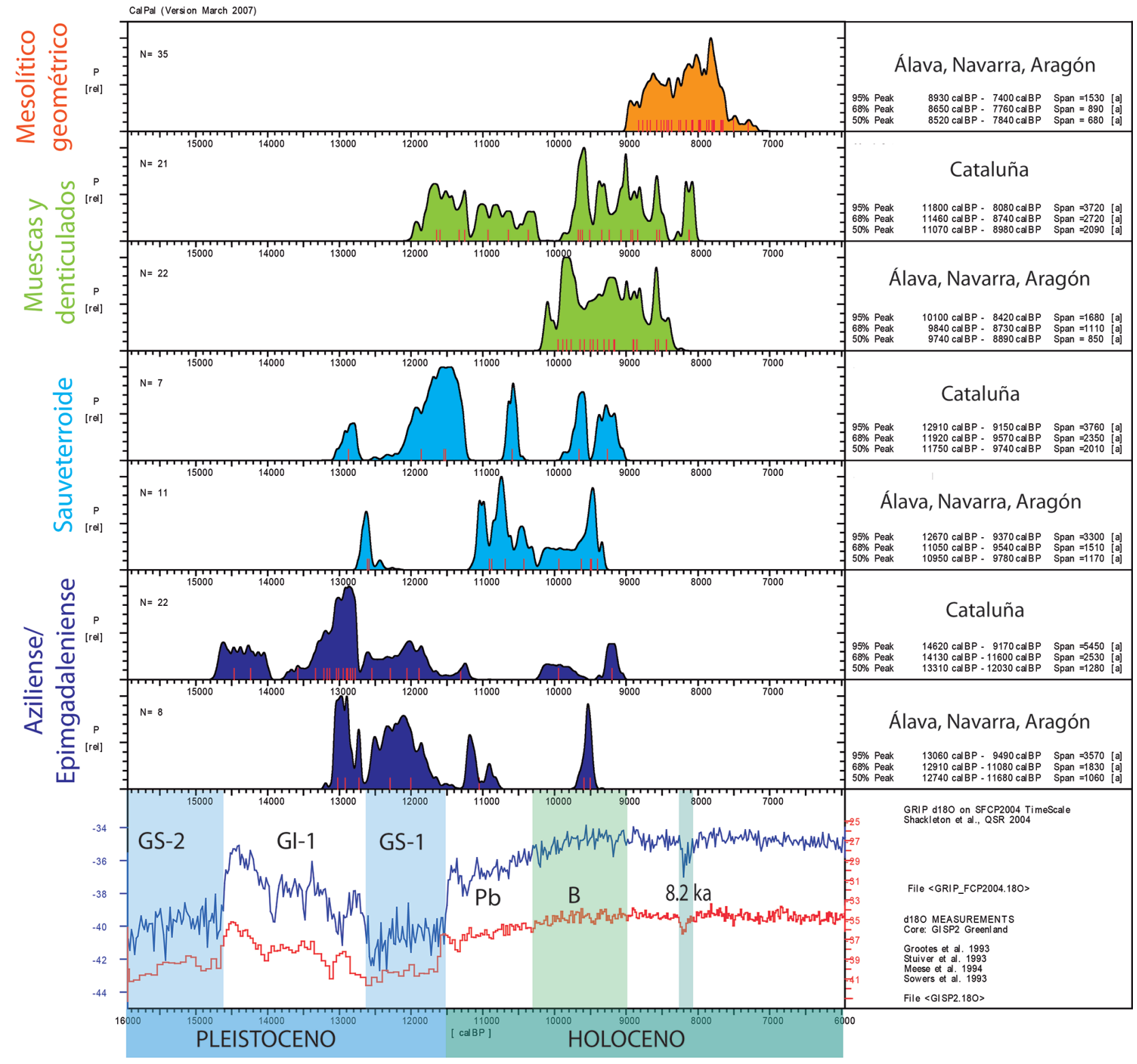

Fig. 2. Evolución cronológica de las tradiciones industriales / Chronological evolution of industrial traditions. 
Aunque cada conjunto responde a dinámicas particulares (según su emplazamiento, funcionalidad y duración de las ocupaciones) sus industrias presentan unos modos comunes de gestión y transformación.

Las estrategias de abastecimiento de las materias primas reflejan la acentuación del proceso de regionalización anunciado en el MSF. Hay un descenso o abandono de los recursos más distantes primando la explotación de los locales. Parco ofrece un ejemplo notable: el descenso de la movilidad o de los intercambios a larga distancia observado en el MSF se intensifica, siendo anecdóticos los recursos foráneos, incorporados como productos finalizados (MANGADO et al., 2005). También en los casos de Mendandia (TARRIÑNO, 2006), Las Orcillas (FERNÁNDEZ-ERASO et al., 2010) o Atxoste se aprecia el predominio de lo local, aunque en éste último las variedades más distantes se introducen en forma de núcleos, participando en la producción (SOTO, 2014). Esta estrategia también supone la incorporación, aunque todavía anecdótica, de rocas de peor calidad, como en Molí del Salt (VAQUERO, 2004), Balma Guilanyà, Balma Margineda (MARTZLUFF, et al., 2012) o La Cativera (MORALES et al., 2013).

Los intereses productivos y las sistemáticas de talla presentan también rasgos comunes. Los soportes laminares siguen siendo el objetivo principal, manteniendo la producción de lascas un menor protagonismo. A pesar de que se mantiene una dualidad productiva -láminas y laminitas- los procesos se simplifican y se acentúa la microlitización.

En la producción de soportes laminares destacan dos procedimientos: a) explotaciones unipolares con escasas acciones de acondicionamiento, aprovechando las características morfológicas de las matrices. La dinámica extractiva suele ser frontal y/o semi-envolvente. Ocasionalmente se recurre a un segundo plano de percusión opuesto para el acondicionamiento. Son raras las explotaciones bipolares -en Atxoste (SOTO, 2014), Filador (DOMĖNECH, 1998) o Molí del Salt (GARCÍA CATALÁN et al., 2013)-; b) producción de laminitas a partir de extracciones unipolares sobre lasca, aprovechando su filo como arista guía, realizando cortas series extractivas frontales o envolventes. Se han reconocido percusiones duras y blandas con percutor orgánico o piedra blanda. Los soportes obtenidos son poco regulares, aproximándose en algunos casos a lascas-laminares.

Estos rasgos son compartidos en las series epipaleolíticas, tanto del extremo occidental, Portugain (AGUIRRE, 2008), Urratxa III (GONZÁLEZ URQUIJO e IBÁÑEZ, 1997), Berniollo (GONZÁLEZ URQUIJO e IBÁÑEZ, 1991) o Atxoste VIb2 (SOTO, 2014), como en el oriental, Parco (MANGADO et al., 2005), Balma Guilanyà (CASANOVA et al., 2007), Molí del Salt (GARCÍA CATALÁN et al., 2013), Filador (DOMĖNECH, 1998) y La Cativera (MORALES et al., 2013). A pesar de los particularismos subyace una concepción común en la relajación de los criterios anteriores de estandarización y regularidad laminar, ejerciendo un menor control en las explotaciones.
Este proceder, así como la microlitización, se han vinculado al reajuste de las estrategias de abastecimiento: el empleo de materiales locales implicaría, en algunos contextos, el uso de nódulos de menor calidad y tamaño, favoreciendo producciones de laminitas (MANGADO et al., 2014; MORALES et al., 2013). Sin embargo, en regiones donde las materias primas no imponen estas limitaciones, como el alto Ebro, plantean la consideración de estos rasgos no como consecuencia, sino como estrategia deliberadamente adoptada dentro de los nuevos modelos de organización económica que supone el Epipaleolítico (SOTO, 2014)

La producción de lascas parece enmarcarse en criterios semejantes (CASANOVA et al., 2007; MARTZLUFF, 2009; GARCÍA CATALÁN et al., 2013; MORALES et al., 2013; SOTO, 2014). La sistemática más habitual, junto a la presencia de núcleos multipolares, es la explotación discoide sobre nódulos o/y lascas. La simplificación de los procedimientos se refleja asimismo en las explotaciones bipolares apoyadas de Balma Margineda y Balma Guilanyà (MARTZLUF et al., 2012).

El utillaje retocado está dominado por el binomio raspador/dorso: los primeros, cada vez más cortos, sobre soportes lascares cuando, en general, las demás categorías usan bases laminares. En los segundos se generalizan los apuntados, frente a la mayor incidencia de las laminitas en el periodo previo y son significativos los cambios en su configuración: habituales retoques bipolares en dorsos espesos, frente a los marginales y unipolares anteriores; mayor incidencia de las morfologías curvas, sin llegar a sustituir a los tipos rectilíneos y variabilidad dimensional, señalándose una progresiva microlitización (Fig.3). El resto del utillaje está constituido por denticulados junto a raederas y truncaduras. Los buriles apenas tienen representación, situación probablemente relacionada con el descenso de la artesanía ósea y los cambios en el equipamiento de caza.

\section{4.- LOS CONJUNTOS SAUVETERROIDES}

Se vienen clasificando como sauveterroides los conjuntos microlaminares con predominio de dorsos donde se reconocen microlitos geométricos, que por sus tipos y dimensiones se asocian al geometrismo que inaugura el Mesolítico al norte de los Pirineos, el Sauveterriense. No obstante se observa un distanciamiento entre ambos geometrismos, siendo destacable en el ibérico: a) su menor protagonismo relativo -no llegan a sustituir a los dorsos-; y b) su mayor antigüedad. Estas diferencias, y principalmente la segunda, plantean cuestiones de difícil respuesta en torno a su origen y vinculación (GARCÍA-ARGÜELLES et al., 2014).

Este sauveterroide arrancaría a finales del Allerød, hacia el 13000-12500, en Parco, Peña-14 y Socuevas (Tabla 1 y Fig.2). Estos comienzos y su desarrollo coinciden en buena medida con el grueso de los conjuntos azilienses y epimagdalenienses, produciéndose en el Dryas III y el Preboreal las ocupaciones de Filador, At- 
xoste o Can Sadurní; y en el Boreal con mayores reservas en Martinarri, El Esplugón, Berroberria y Marge del Moro, advirtiendo en estos dos últimos un fuerte componente de utensilios propios del MMD. Se sumarían las colecciones, datadas pero con amplias desviaciones, de Balma Margineda y Balma del Gai.

A pesar del solapamiento temporal del Aziliense/Epimagdaleniense y del Sauveterroide, en las secuencias estratigráficas la segunda tradición se superpone a las primeras (Parco, Filador, Balma del Gai, Balma Margineda, Can Sadurní, Socuevas, Atxoste) (Tabla 1 y Fig.1), sin que por ello deje de ser compleja la explicación de esta contemporaneidad que se da en todo el territorio.

Industrialmente no parece producirse una ruptura evidente con respecto a la tradición previa, siendo extrapolables las características señaladas. El elemento diagnóstico son los geométricos, cuyos tipos más habituales son triángulos isósceles y escalenos de pequeñas dimensiones, llegando en ocasiones a ser hipermicrolíticos (SOTO, 2014), junto a segmentos. El peso de este geometrismo es cuantitativamente débil (5-15\% de lo retocado), sin llegar a sustituir el predominio de los dorsos. Asociados a su configuración se reconoce la técnica del microburil.

Siendo éste el rasgo más discriminante del sauveterroide, se van reconociendo otros cambios en la configuración del armamento. Así, en Atxoste y Peña-14 se acentúa la microlitización y pérdida de robustez de los dorsos con respecto a la fase previa (SOTO et al., 2015), mientras que en Balma Margineda junto a los geométricos, cobran un notable protagonismo puntas de truncadura muy oblicua (MARTZLUFF, 2009).

De forma incipiente y sin poder evaluar su entidad, se reconocen otras transformaciones en el ámbito del proceso productivo. En Margineda y Parco hay una acentuación de la regionalización en el abastecimiento de las materias primas, incorporando nuevos recursos locales (MARTZLUFF, 2009; MANGADO et al., 2014). Sin embargo, en Atxoste los cambios no afectan tanto al abastecimiento como a su gestión, apreciándose un aprovechamiento más intensivo (microlitización y mayor incidencia de reciclajes). Asimismo, se abandonan ciertas sistemáticas laminares, como la bipolar, a favor de esquemas unipolares más cuidados (SOTO, 2015), similar a lo señalado en Filador (DOMĖNECH, 1998).

\section{5.- EL MESOĹTICO DE MUESCAS Y DENTICULADOS}

Cronológicamente, el arranque del Mesolítico de muescas y denticulados presenta notables diferencias en el territorio, con un desfase de unos dos milenios entre los extremos oriental y occidental (Fig.2). En Cataluña las dataciones se agrupan en dos bloques: el primero entre el 12000-10300, con las ocupaciones del Abric Agut, Balma Guilanyà y Picamoixons, en coincidencia con una parte de los registros sauveterroides y del Aziliense/Epimagdaleniense (Fig.3). El segundo arranca hacia el 10300, iniciando con el Boreal su desarrollo en el resto del territorio: en el occidente Mendandia, Kanpanoste Goikoa, Kanpanoste, Atxoste y Fuente $\mathrm{Hoz}$; en el área pirenaica navarra Berroberría, Aizpea y Artusia; en Aragón Peña-14, Legunova, Forcas-II, Los Baños, Ángel-1, Ángel-2, Pontet, Costalena y Plano del Pulido; en Cataluña se unen Balma Margineda, Font del Ros, Roc del Migdia, Sota Palou, Filador, La Cativera, Molí del Salt, Can Sadurní, Marge del Moro y Balma del Serrat del Pont. Esta tradición finaliza hacia el 8500, ampliándose hasta el 8000 -en coincidencia con el inicio del MG- según las informaciones preliminares de Can Sadurní (FULLOLA et al., 2011) y de Balma del Serrat del Pont, con las reservas planteadas (VAQUERO y GARCÍA-ARGÜELLES y GARCÍA-ARGÜELLES, 2009).

El modelo de ocupación territorial y las pautas económicas no varían. Son bastantes los sitios sin solución de continuidad en su ocupación (Atxoste, Guilanyà o Margineda), aunque tampoco faltan ejemplos de abandonos (Parco, Legunova, Peña-14, Martinarri o Socuevas) así como otros de nueva creación (Ángel, Font del Ros, Kanpanoste, Sota Palou...), destacando dos situaciones de especial interés: a) la confirmación de un poblamiento al aire libre; y b) la distribución por una más amplia área geográfica, incluyendo ahora la margen derecha del Ebro (Fig.1).

Sin perder de vista estas continuidades, el sistema lítico presenta cambios profundos que evidencian una organización de la producción y gestión de la industria lítica, así como probablemente una funcionalidad, distinta a las imperantes en los momentos previos.

Respecto a las materias primas continúa la tendencia a la reducción de los territorios de captación. En aquellos yacimientos con ocupaciones epipaleolíticas previas, se hace patente el incremento de los recursos locales. Esto supone en la mayoría de los casos la desaparición o rarificación de los materiales de procedencia distante, así como un cambio en la intensidad o en la naturaleza de las relaciones con las regiones de procedencia de dichos materiales. Esta dinámica implica el empleo de recursos locales, aunque suponga la incorporación de sílex de mediocre calidad o de otro tipo de rocas, que a pesar de sus limitaciones para la talla, responden con suficiencia a las nuevas prácticas tecnológicas. El incremento de estos materiales se registra en Margineda (MARTZLUFF et al., 2012), Guilanyà (CASANOVA et al., 2007), La Cativera (VAQUERO, 2006), Molí del Salt (VAQUERO, 2004), Mendandia (TARRIÑO, 2006), Atxoste (SOTO, 2014) o en Biel (MONTES et al., 2016), y se reproduce en yacimientos de nueva fundación, como Font del Ros (TERRADAS, 1995), Roc del Migdia (RODRíGUEZ e YLL, 1991), Sota Palou (MARTÍNEZ-MORENO et al., 2006-2007), Abric Agut (VAQUERO, 2006) o Kanpanoste (TARRIÑO, 2004).

El aprovechamiento de materiales con restricciones en su calidad es posible por el cambio profundo en los intereses productivos, destacando el abandono 


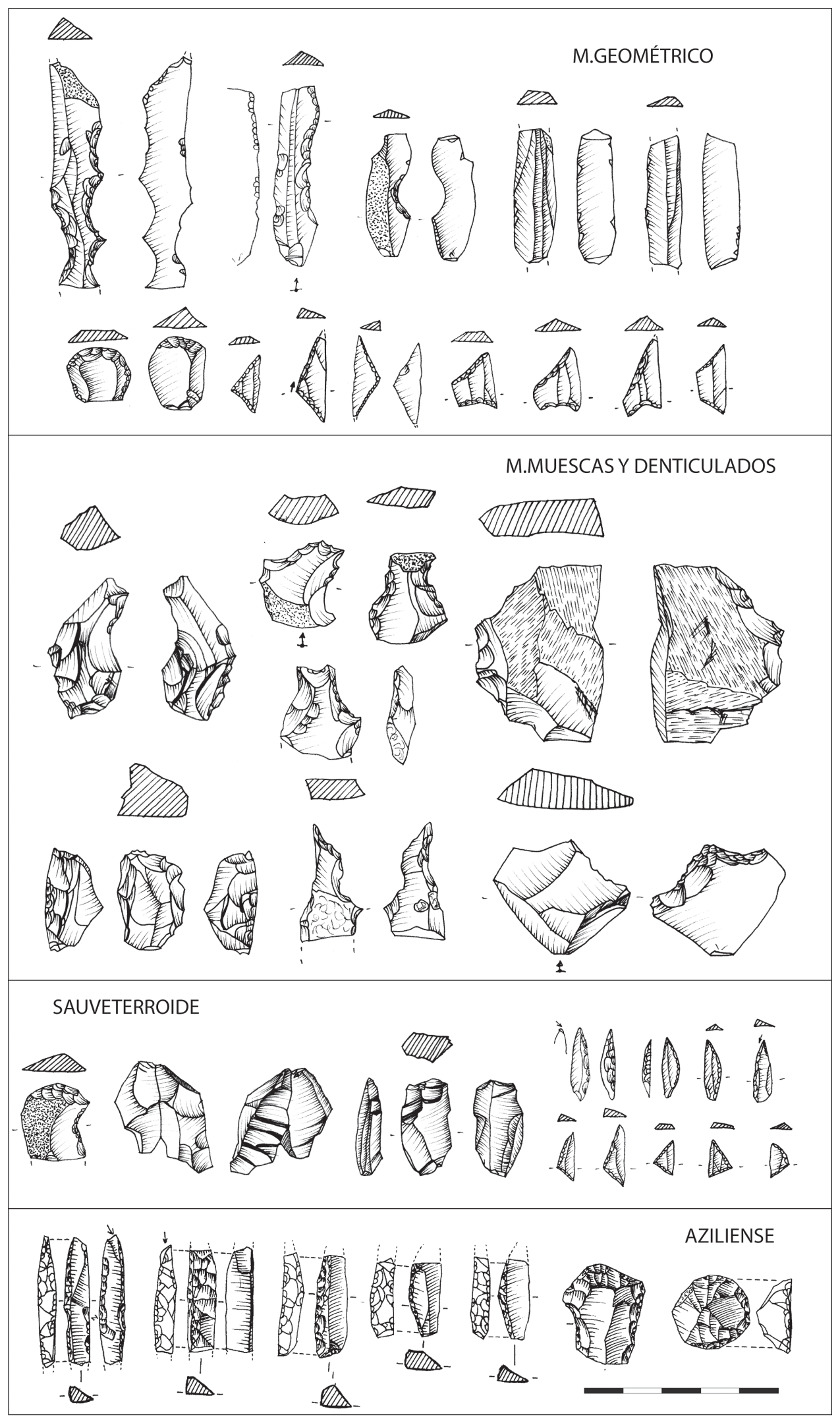

Fig. 3. Esquema de la industria retocada del Epipaleolítico y Mesolítico (dibujos de la secuencia de Atxoste -SOTO, 2014- excepto los del Aziliense, provenientes de Portugain -CAVA, 2008-) / Epipaleolithic and Mesolithic retouched industry scheme (all drawing are from Atxoste -SOTO,2014- excepted Azilian examples, from Portugain -CAVA, 2008-). 
o marginalidad de la producción laminar (3-20\% según contextos). Se desarrolla mediante sistemáticas sencillas, generalmente unipolares, sin acciones de acondicionamiento, obteniendo series cortas de productos poco estandarizados próximos a lascas-laminares.

El objetivo principal es la obtención de lascas, aplicándose generalmente tres sistemáticas: a) explotaciones unipolares sobre una o varias superficies de talla, que se convierten en multipolares al cambiar la función de los planos de trabajo cuando las condiciones no son aptas para su continuidad; b) explotaciones discoides unifaciales o bifaciales sobre lascas y nódulos (MARTZLUFF et al., 1995; TERRADAS, 2003; CASANOVA et al., 2007; VAQUERO, 2006; SOTO, 2014) sea para procedimientos cortos y expeditivos o para dinámicas más intensivas con acciones de acondicionamiento; y c) explotaciones bipolares apoyadas, vinculadas a materias primas de escasa calidad, nódulos de pequeñas dimensiones y lascas espesas, aprovechando recursos que de otro modo probablemente serían desechados (MARTÍNEZ-MORENO et al., 2006-2007; MARTZLUFF et al., 1995; CAVA, Cava, 2004a; SOTO, 2014) (Fig.4).

Es común la definición de estas dinámicas como a) expeditivas, por su escaso acondicionamiento, aprovechando las morfologías naturales de las matrices, y b) poco productivas, por el abandono de los núcleos tras la extracción de un número reducido de lascas (ALDAY, 2006). No obstante, y aunque es pronto para su correcta evaluación, en algunas secuencias como Atxoste (SOTO, 2014) o Balma Margineda (MARTZLUFF et al., 1995), se reconocen explotaciones más intensivas y cuidadas, denotando un mejor aprovechamiento de los recursos así como un mayor control sobre las características finales de los productos.

Este cambio en las estrategias productivas va en consonancia con la transformación del utillaje retocado, tanto en su composición como configuración. Destaca especialmente el descenso muy acusado o abandono de la producción de proyectiles, apenas alcanzando el $1-2 \%$ de las colecciones. En cambio, los elementos de frentes denticulados (raederas, perforadores, muescas y denticulados, dependiendo de los criterios clasificatorios seguidos) llevan el peso de las producciones. Los estudios traceológicos (MAZO, 2006; RODRÍGUEZ, 1993) observan, por el momento, lo que era poco más que una intuición: buena parte de este utillaje se vincula al trabajo sobre materias orgánicas, pudiendo asociarse a la fabricación de un nuevo armamento, sustituyendo al realizado en piedra.

Estas piezas se realizan sobre soportes poco estandarizados, empleando además de lascas, trozos, productos de acondicionamiento o núcleos amortizados. Esta selección aparentemente aleatoria, formaría parte de un programa complejo para el aprovechamiento intensivo de los recursos disponibles, al menos en el alto Ebro (Cava, 2004a, 2006; SOTO, 2014). Este programa, a tenor de lo observado en el caso de Atxos- te, se fundamentaría asimismo en un cambio en los criterios tecnológicos de configuración, permitiendo una gestión más versátil del utillaje, mediante el desarrollo de reavivados y reciclajes, que explicarían en buena medida, algunas particularidades morfológicas de estas industrias (combinación de múltiples filos retocados en un mismo objeto; retoques escamosos y profundos con disposiciones inversas; pequeñas dimensiones pero carácter carenado de las piezas...) (SOTO, 2014) (Fig.4). No obstante, será necesario contrastar estas estrategias en otros lugares, ya que no descartamos que se plantearan distintas situaciones de gestión, de acuerdo a la disponibilidad de materiales y sus posibilidades. Así, en conjuntos catalanes, como Font del Ros o Roc del Migdia, se identifican piezas de mayores dimensiones y características configurativas diferentes, que no necesariamente se ajustarían a los criterios de gestión observados en el alto Ebro.

En todo caso, las dinámicas adoptadas en los distintos ámbitos del sistema lítico están bien articuladas entre sí, construyendo una estrategia organizativa propia y diferente a las contemporáneas del occidente europeo. El aprovechamiento de los recursos locales, incluyendo materiales de regular calidad, es posible con estos patrones productivos poco rígidos y de gestión dinámica, donde la concepción tecnomorfológica contrasta con las tradiciones anteriores: a objetivos distintos le corresponden diferentes procedimientos. Lejos queda la consideración de estas industrias como coyunturales, reconociéndose su desarrollo como fruto de unas estrategias polivalentes (ALDAY, 2006; MARTÍNEZ-MORENO et al., 2006-2007) de mayor complejidad organizativa que la considerada inicialmente (SOTO, 2014).

\section{EL MESOLÍTICO GEOMÉTRICO}

La anterior realidad industrial conoce un cambio profundo con el desarrollo de los conjuntos del Mesolítico geométrico. Aunque los rasgos de las industrias de muescas y denticulados no desaparecen de manera brusca, de hecho varios se mantienen en secuencias del primer geometrismo tardenoide (ALDAY y CAVA, 2009; UTRILLA et al., 2009), la recuperación de la producción laminar y de los proyectiles en piedra afecta profundamente a la organización del sistema lítico.

Se desarrollan estrategias planificadas en relación a la gestión de la materia prima y al sistema de producción. La manufactura laminar se desarrolla bajo parámetros de gran regularidad y estandarización, exigiendo calidad en las materias primas. Así, en el alto Ebro, aunque se mantiene el carácter local del abastecimiento, se proveen de materiales de alta calidad, desarrollándose gestiones diferenciadas según las variedades silíceas (CAVA et al., 2007-2008). Se priorizan sistemáticas unipolares aplicando distintos procedimientos para el mantenimiento y control de la regularidad de la producción, identificándose en algunos casos la percu- 


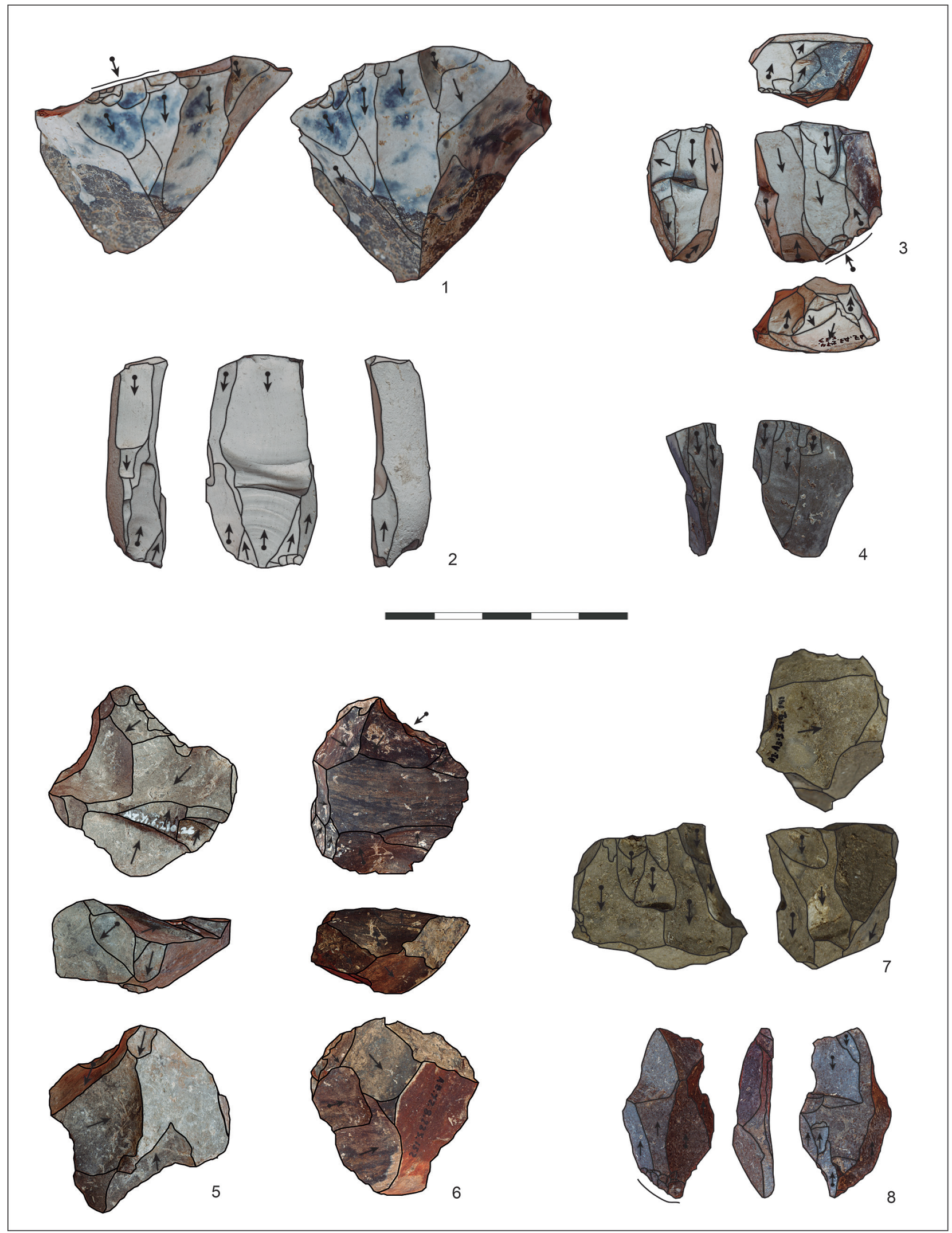

Fig. 3. Ejemplos de núcleos del Epimagdaleniense (1- unipolar convergente; 2- bipolar); del Sauveterroide (3- Unipolar con plano de percusión opuesto secundario; 4- unipolar sobre arista de lasca); y del Mesolítico de muescas y denticulados (5 y 6- discoides; 7- unipolar; 8- bipolar apoyada) de Atxoste (SOTO, 2014) / Cores examples from the Epimagdalenian, Sauveterroid and notches and denticulate Mesolithic from Atxoste. 
sión indirecta (SOTO, 2014). Esta regularidad laminar se relaciona con las exigencias del nuevo armamento, formado mediante el ensamblaje de dorsos y microlitos geométricos. La elaboración de trapecios y triángulos son sus señas de identidad: habiéndose detallado suficientemente sus evoluciones y particularismos regionales (ALDAY y CAVA, 2009; UTRILLA et al., 2009) nos contentamos aquí con señalar que es un equipamiento de dimensiones y tipología diferentes a los sauveterroides. Son abundantes también las raederas y los raspadores, a la vez que el empleo de láminas en bruto irá, progresivamente, tomando protagonismo (Fig.4).

Esta tradición industrial conecta a la región con las contemporáneas del occidente europeo, pues estamos ante un proceso de renovación de gran amplitud geográfica (PERRIN et al., 2009; BINDER et al., 2012). No obstante, en las tierras más orientales, en Cataluña, no se conocen evidencias arqueológicas claras de esta unidad: se mencionan algunos casos poco explícitos (VAQUER y GARCÍA-ARGÜELLES, 2009) de manera que los yacimientos con dataciones que encajarían en los inicios del episodio presentan secuencias propias del Mesolítico de muescas y denticulados, mientras que para la primera mitad del séptimo milenio carecemos de documentación. Posibles cambios en los patrones del poblamiento o procesos erosivos son algunas de las hipótesis esgrimidas para la explicación de este hecho, siendo cuestiones por el momento difícilmente contrastables (lbíd.).

\section{REFLEXIÓN FINAL}

Los notables avances realizados en la investigación sobre el Epipaleolítico y el Mesolítico en la vertiente sur de los Pirineos permiten disponer de un conocimiento significativo sobre las distintas estrategias de organización y desarrollo de las industrias líticas. Uno de los aspectos más reseñables es la notable homogeneidad que muestra el territorio en su evolución industrial. Aunque se ha reconocido una progresiva regionalización en las dinámicas de abastecimiento de los recursos líticos desde el MSF, ejemplarizando una movilidad más acotada, los contactos entre las distintas áreas surpirenaicas continúan vigentes participando de los mismos cambios tecnológicos, probablemente a través de distintas redes de intercambio (MONTES y ALDAY, 2012; MARTÍNEZ-MORENO et al., 2010). No obstante, ello no impide la identificación incipiente de particularismos regionales, probablemente sujetos a las distintas realidades contextuales que se dan en un territorio tan extenso y diverso. Así, está pendiente: la evaluación de la entidad cultural -no sólo industrial- del Aziliense y del Epimagdaleniense o Epipaleolítico microlaminar, ¿en qué medida constituyen realidades diferentes?; la profundización en la mayor diversidad de los conjuntos del MMD; o el desafío que supone la ausencia de registros del MG en Cataluña.

Más allá de estas cuestiones, el objetivo perseguido ha sido poner en evidencia estrategias compartidas por aquellas sociedades cazadoras-recolectoras entre finales del Tardiglaciar y la llegada del Neolítico. En este sentido, destaca la variedad de tradiciones desarrolladas y la relativa rapidez con la que se suceden, ante unos modos de vida que aparentemente no cambian significativamente. Ante esta realidad, los nuevos retos que se plantean deben girar necesariamente en torno a la comprensión de los mecanismos y causas que motivan estas transformaciones industriales, siendo necesaria su vinculación con otros ámbitos sociales.

Así, las modificaciones industriales que arrancan en el MSF y que se generalizan en el Epipaleolítico se producen en relación a cambios en la movilidad, en la economía e incluso en el ámbito simbólico. Las novedades en el armamento, con la generalización de las puntas dorso, o la tendencia a la simplificación y microlitización de la producción, son transformaciones progresivas, de difícil explicación socio-histórica, pero que constituyen una forma diferente de concebir y gestionar el material lítico, sujetas a la nueva realidad postpaleolítica. En estas tendencias de largo recorrido, los conjuntos sauveterroides aportan novedades no solo en el armamento, sino que las transformaciones afectan también a la organización del sistema lítico, sin que los cambios supongan tanto una ruptura con la fase anterior como una evolución interna.

En cambio, la ruptura industrial viene de la mano del MMD, al finalizar con la línea inspirada desde el MSF, pero también ruptura geográfica (MARCHAND, 2014), al romper con las tendencias industriales del occidente europeo, aunque ambas premisas deben matizarse. Primero porque algunos de los rasgos que definen estas industrias empiezan a manifestarse en las tradiciones anteriores (reducción territorial, simplificación en los procesos...) (MARTíNEZ-MORENO et al., 2006-2007; CAVA, 2004b). Segundo porque en la Europa continental se conocen algunas situaciones que al menos en parte coinciden con las ibéricas (FRANCO, 2011; MARTINI, 2005; PERLÈS, 1990; ESCALON DE FOTON 1976; LAPLACE, 1953) planteando interesantes cuestiones sobre la evolución del Mesolítico de Europa occidental. En este sentido, el MG implica una nueva etapa, anclándonos de nuevo en las dinámicas europeas, participando de un cambio general (PERRIN et al., 2009; BINDER et al., 2012). En nuestro territorio las novedades tecnológicas no suponen una ruptura industrial con lo anterior, ya que en sus primeras fases se mantiene, de manera oscilante en cada caso, rasgos propios del MMD, aunque conlleva otras dinámicas, como la ausencia de documentación en Cataluña, de más compleja explicación.

\section{AGRADECIMIENTOS}

El presente texto se enmarca en el trabajo realizado en las redes de investigación CTP PREHISTOPYR y PALMESOPYR, así como en los proyectos HAR201459042 (Transiciones climáticas y adaptaciones sociales 
en la Prehistoria de la Cuenca del Ebro) y HAR201455131, y en el Grupo de Investigación del Gobierno de Aragón H07-PPVE (Primeros Pobladores del Valle del Ebro) y con el soporte del IUCA (Instituto Universitario de Ciencias Ambientales).

\section{BIBLIOGRAFÍA}

AGUIRRE, M.

2008 Caracterización tecnológica de la industria de Portugain, en BARANDIARÁN, I. y CAVA, A. Cazadores y tallistas en el abrigo de Portugain. Una ocupación de Urbasa durante el Tardiglaciar. Fundación José Miguel de Barandiarán. Vitoria-Gasteiz, 121-183.

\section{ALDAY, A.}

1998 Kanpanoste Goikoa. El depósito prehistórico de Kanpanoste Goikoa (Vírgala, Álava): memoria de las actuaciones arqueológicas 1992-1993. Vitoria: Memorias de Yacimientos Alaveses 5.

$2006 a$ El campamento prehistórico de Mendandia: ocupaciones mesolíticas y neolíticas entre el 8800 y el 6400 B.P. Memorias Arqueología en Castilla y León. Junta de Castilla y León.

2006b El Mesolítico de muescas y denticulados en la cuenca del Ebro y el litoral mediterráneo peninsular: síntesis de Ios datos, en ALDAY, A. El Mesolítico de muescas y denticulados en la cuenca del Ebro y el litoral mediterráneo peninsular, Memoria de yacimientos Alaveses, 11, 303-317.

2014 El yacimiento de Atxoste (Vírgala, Álava), en SALA, R. (ed.) Los cazadores recolectores del Pleistoceno y del Holoceno en Iberia y el estrecho de Gibraltar. Estado actual del conocimiento del registro arqueológico. Universidad de Burgos, 148-151.

ALDAY, A. y CAVA, A.

2009 El Mesolítico geométrico en Vasconia, en UTRILLA, P. y MONTES, L. (eds.). El Mesolítico Geométrico en la Península Ibérica. Monografías Arqueológicas. Prehistoria. Universidad de Zaragoza. Zaragoza, 93-129.

2010/11 Yacimiento prehistórico de Socuevas (Pobes). Campañas. Arkeoikuska, 2009 y 2010.

ALDAY, A.; SOTO, A.; LÓPEZ DE HEREDIA, J. y PERALES, U.

2012 El abrigo de Martinarri (Obécuri, Treviño): una ocupación del Tardiglaciar en la cuenca alta del Ebro. Trabajos de Prehistoria, 69, 2, 257-272.

\section{ARMENDÁRIZ, A.}

1997 Anton Koba: Cazadores azilienses en la Sierra de Aizkorri (Gipuzkoa), en BALBíN, R. y BUENO, P. I/ Congreso de Arqueología Peninsular. Tomo I. Paleolítico y Epipaleolítico. Fundación Rei Afonso Henriques, 297-310.

BALDEÓN, A., BERGANZA, E. y GARCÍA, E.

1983a Estudio del yacimiento de El Montico de Charratu (Albaina, Treviño). Estudios de Arqueología Alavesa, $\mathrm{n}^{\circ}$ 11, 121-186.
BALDEÓN, A., GARCÍA, E., ORTIZ, I., LOBO, P.

1983b Excavaciones en el yacimiento de Fuente Hoz (Anúcita, Álava). Estudios de Arqueología Alavesa, 11, 7-68.

\section{BARANDIARÁN, I.}

1993/94 Cueva de Berroberría (Urdax). Informe de las campañas de excavación V (1990), VI (1991), VII (1992) y VIII (1993). Trabajos de Arqueología Navarra, 11, 243-247.

BARANDIARÁN, I. y CAVA, A.

2000 A propósito de unas fechas del Bajo Aragón: Reflexiones sobre el Mesolítico y el Neolítico en la cuenca del Ebro. SPAL, 9, 293-326.

2001a El Paleolítico superior de la cueva de Zatoya (Navarra): actualización de los datos en 1997. Trabajos de Arqueología Navarra, 15, 5-100.

2001b Cazadores-recolectores en el Pirineo navarro: el sitio de Aizpea, entre 8.000 y 6.000 años antes de ahora. Universidad del País Vasco, Euskal Herriko Unibertsitatea ed. Vol. 10. Vitoria-Gasteiz.

2008 Cazadores y tallistas en el abrigo de Portugain. Una ocupación de Urbasa durante el Tardiglaciar. Fundación José Miguel de Barandiarán. Vitoria-Gasteiz.

\section{BARANDIARÁN, I., CAVA, A. y ALDAY, A.}

2006 Ocupaciones de altura e interior durante el Tardiglaciar: la Llanada alavesa y sus estribaciones montañosas. Zona Arqueológica, 7. Miscelánea en homenaje a Victoria Cabrera, 534-551.

BINDER, D., COLLINA, C., GUILBERT, R., PERRIN, T. y GARCIA-PUCHOL, O

2012 Pressure-Knapping Blade Production in the North-Western Mediterranean Region During the Seventh Millennium cal B.C., en DESROSIERS, P. M. (ed.), The Emergence of Pressure Blade Making. From Origin to Modern Experimentation. Springer, 199-217.

$\mathrm{BOSCH}, \mathrm{J}$.

1993 Cronologia prehistòrica al curs inferior de l’Ebre. Primeres datacionsn absolutes. Pyrenae, 24, 53-56.

\section{CASANOVA, J., MARTÍNEZ-MORENO, J. y MORA, R.}

2007 Traçant l'ocupació dels Pirineus: la Balma Guilanyà i els caçádors recollectors del Tardiglacial i l'Holocè antic al Prepirineu oriental. Tribuna d'Arqueologia, 59-83.

CAVA, A.

2004a La industria lítica de Kanpanoste (Vírgala, Álava), en CAVA, A. La ocupación prehistórica de Kanpanoste en el contexto de los cazadores-recolectores del Mesolítico. Memoria de Yacimientos Alaveses, 9.

2004b Los procesos culturales del comienzo del Holoceno en la cuenca del Ebro y su contextualización. Salduie, 4, 17-40.

2008 La industria lítica de Portugain: los objetos retocados, descripción y reflexiones tecno-tipológicas, en BARANDIARÁN, I. y CAVA, A. Cazadores y tallistas en el abrigo de Portugain. Una ocupación de Urbasa durante el Tardiglaciar. Fundación José Miguel de Barandiarán. Vitoria-Gasteiz, 59-119. 
CAVA, A y BEGUIRISTAIN, M. A.

1991/92 El yacimiento prehistórico del abrigo de la Peña (Marañón, Navarra). Trabajos de Arqueología Navarra,10, 69-116.

CAVA, A., ALDAY, A. y TARRIÑO, A.

2007/08 La circulación de materia primas líticas en la transición Mesolítico/Neolítico antiguo en el País Vasco. Los abrigos de Mendandia, Kanpanoste y Aizpea. Veleia, 24 25. Homenaje a Ignacio Barandiarán Maestu, 581-609.

\section{DOMÈNECH, E.}

1998 Los sistemas de producción lítica del Paleolítico Superior final y Epipaleolítico en la vertiente mediterránea occidental. Pyrenae, 29, 9-45.

DOMINGO, R. y MONTES, L.

2009 Valcervera y Rambla de Legunova: dos yacimientos postpaleolíticos en Biel, Zaragoza Valcervera and Rambla de Legunova. Salduie, 9, 295-310.

\section{ESCALON DE FOTON, M.}

1976 Les civilisations de l'Epipaléolithique et du Mésolithique en Provence, La Préhistoire en France I. Les civilisations paléolithiques et mésolithiques de la France, 1367-1378.

FERNÁNDEZ ERASO, J., GARCÍA ROJAS, M., FERNÁNDEZ CRESPO, T., CASTAÑOS, P., BAILON, S., MURELAGA, X. y TARRIÑO, A.

2010 La cueva de Las Orcillas 1: una estación de los últimos cazadores-recolectores en La Berrueza (Mendaza-Acedo, Navarra). Trabajos de Arqueología Navarra, 22, 13-91.

FORTEA, J.

1973 Los complejos microlaminares y geométricos del Epipaleolítico mediterráneo español, Salamanca.

FRANCO, C.

2011 La fine del Mesolitico in Italia: identità culturale e distribuzione territoriale degli ultimi cacciatori-raccoglitori. Società per la preistoria e protostoria della Regione Friuli Venezia Giulia, Quaderni 13.

FULLOLA, J.M., BARTROLÍ, R., BERGADÀ, M., DOCE, R., GARCIA-ARGUUEELLES, P., RODON, T., ADSERIAS, M. y CEBRIÀ, A.

1993 Nuevas aportaciones al conocimiento del Paleolítico Superior en las comarcas meridionales y occidentales de Cataluña, en FUMANAL, M. P y BERNABEU, J. (eds.), Estudios sobre Cuaternario. Medios sedimentarios. Cambios ambientales. Hábitat humano, Universitat de València, València, 239-247.

FULLOLA, J., GARCíA-ARGÜELLES, P., MANGADO, X. y MEDINA, B.

2011 Paleolític i Epipaleolític al Garraf-Ordal. On érem i on somn, en BLASCO, A., EDO, M. y VILLALBA, M.J. La cova de Can Sadurní i la Prehistòria de Garraf. Recull de 30 anys d'investigació. EDAR, Arqueología y Patrimonio, 227-243.
GARCÍA-ARGÜELLES, P., ESTRADA, A., FULLOLA, J. M., NA$D A L$, J. y MANGADO, $X$.

2009 Les niveaux épipaléolithiques de la Balma del Gai (Moià, Barcelone, Catalogne), en Méditerranée et D'ailleurs: Mélanges Offerts À Jean Guilaine, Archives d'Ecologie préhistorique, 299-310.

GARCÍA-ARGÜELLES, P., FULLOLA, J. M., ROMÁN, D., NADAL, J. y BERGADÀ, $M^{\mathrm{a}} \mathrm{M}$

2013 El modelo epipaleolítico geométrico tipo Filador cuarenta años después: vigencia y nuevas propuestas, en DE LA RASILLA, M. Homenaje a Javier Fortea Pérez. Universidad de Oviedo. Ménsula Ediciones, 151-165.

GARCÍA-ARGÜELLES, P., NADAL, J., FULLOLA, J. M., BERGADÀ, Ma M., DOMINGO, I., ALLUÉ, E. y LLOVERAS, L.

2014 Nuevas interpretaciones del Paleolítico superior fina de la Cataluña meridional: el yacimiento de L'Hort de la Boquera (Priorat, Tarragona). Trabajos de Prehistoria, $71,242-260$

GARCÍA-ARGÜELLES, P., NADAL, J. y FULLOLA, J. M.

2005 El abrigo del Filador (Margalef de Montsant, Tarragona) y su contextualización cultural y cronológica en el nordeste peninsular. Trabajos de Prehistoria, 62, 1, 65-83.

GARCÍA CATALÁN, S., GÓMEZ DE SOLER, B., SOTO, M. y VAQUERO, M

2013 Los sistemas de producción lítica en el Paleolítico superior final: el caso del nivel Asup del Molí del Salt (Vimbodí i Poblet, Tarragona). Zephyrus, LXXII, 39-59.

GARCÍA CATALÁN, S., VAQUERO, M., PÉREZ GOÑI, I., MENÉNDEZ, B., PEÑA GARCÍA, L., BLASCO, R., MANCHA, E., MORENO, D. y MUÑOZ ENCINAR, L.

2009 Palimpsestos y cambios culturales en el límite Pleistoceno-Holoceno: el conjunto lítico de Picamoixons (Alt Camp, Tarragona). Trabajos de Prehistoria, 66, 2, 61-76.

\section{GARCÍA GAZÓLAZ, J.}

2001 Excavaciones arqueológicas en el abrigo del Padre Areso (Bigüézal, Navarra). Campañas de 1994-19951996. Trabajos de Arqueología Navarra, 15, 307-314.

GARCÍA MARTÍNEZ DE LAGRÁN, I., IRIARTEC, E., GARCÍA GAZÓLAZ, J., TEJEDOR, C., GIBAJA, J.F., MORENO GARCIAA, M., PÉREZ JORDÁ, G., RUIZ-ALONSO, M., SESMA, J., GARRIDO, R., CARRANCHO, A., PEÑA CHOCARRO, L. y ROJO, M.

20168.2 ka BP paleoclimatic event and the Ebro Valley Mesolithic groups: Preliminary data from Artusia rock shelter (Unzué, Navarra, Spain). Quaternary International $403,151-173$

GONZÁLEZ URQUIJO, J. E. e IBÁÑEZ, J. J

1991 La tecnología de talla laminar en la ocupación epipaleolítica de Berniollo. (Subijana-Morillas, Álava), en MORA, R., TERRADAS, X., PARPAL, A. y PLANA, F. Tecnología y Cadenas Operativas Líticas. Treballs d'Arqueologia, I, 201-222.

1997 Fabricación de los útiles de piedra en el yacimiento de Urratxa III (Orozko, Bizkaia), en MUÑOZ, M. y BERGANZA, E. El yacimiento de la cueva de Urratxa III (Orozko, Bizkaia). Universidad de Deusto, Bilbao, 123-133. 


\section{GUILAINE, J. y MARTZLUFF}

1995 Les excavacions a la Balma de la Margineda (19791991). Estudi arquèologic (Govern d'A.). Andorra.

\section{LANGLAIS, M.}

2007 Dynamiques culturelles des sociétés magdaléniennes dans leurs cadres environnementaux. Enquêtes sur 7000 ans d'évolution de leurs industries lithiques entre Rhône et Ebre. Tesis doctoral. Université de Toulouse-Le Mirail y Universidad de Barcelona.

\section{LAPLACE, G}

1953 Les couches à escargots des cavernes pyrénéennes et le problème de l'Arisien de Piette. Bulletin de la Société Préhistorique Française, 50, 4, 201-208

MANGADO, X., BARTROLÍ, R., CALVO, M., FULLOLA, J. y PETIT, M ${ }^{2} A$.

2005 Les industries lithiques de la fin du Paléolithique de la Grotte du Parco (Alòs de Balaguer, Catalogne), en J. P. e. M. BRACCO, D'un monde à l'autre. Les systèmes lithiques pendant le Tardiglaciaire autour de la Méditerranée nord-occidentale. Actes de la table ronde internationale. Aix-en-Provence (2001), 11-24.

MANGADO, X., PETIT, M. FULLOLA, J. Ma y BARTROLÍ, R.

2006/07 El Paleolitic superior final de la Cova del Parco (Alòs de Balaguer, La Noguera). Revista d'Arqueologia de Ponent, 16-17, 45-62.

MANGADO, X., TEJERO, J.M., FULLOLA, J. Mª., PETIT, M ${ }^{a}$ A. y SÁNCHEZ, M.

2014 La Cova del Parco (Alòs de Balaguer, La Noguera, Lleida). La secuencia del Magdaleniense, en SALA, R. Los cazadores recolectores del Pleistoceno y del Holoceno en Iberia y el estrecho de Gibraltar: Estado actual del conocimiento del registro arqueológico. Universidad de Burgos y Fundación Atapuerca: 152-160.

\section{MARCHAND, G.}

2014 Premier et second Mésolithique: et au-delà des techniques? en HENRY, A., MARQUEBIELLE, B., CHESNAUX, L. y MICHEL, S. (eds.). Des techniques aux territoires: nouveaux regards sur les cultures mésolithiques. Actes de la table-ronde, 22-23 novembre 2012, Maison de la recherche, Toulouse, Palethnologie, 11-22.

\section{MARIEZKURRENA, K.}

1990 Dataciones absolutas para la arqueología vasca. Munibe, 42, 287-304.

\section{MARTÍNEZ-MORENO, J. y MORA, R.}

2009 Balma Guilanyà (Prepirineo de Lleida) y el Aziliense en el noreste de la Península Ibérica. Trabajos de Prehistoria, 66, 2, 45-60.

\section{MARTÍNEZ-MORENO, J., MORA, R. y CASANOVA, J.}

2006/07 El contexto cronométrico y tecno-tipológico durante el Tardiglaciar y Postglaciar de la vertiente sur de los Pirineos orientales. Revista d'Arqueologia de Ponent, 16-17, 7-44.
2010 Lost in the mountains? Marine ornaments in the Mesolithic of the northeast of the Iberian Peninsula, en ÁLVAREZ-FERNÁNDEZ, E. y CARVAJAL, D. (eds.) Not only food. Marine, terrestrial and freshwater molluscs in archaeological sites. Munibe, suplemento 31, 100-109.

MARTINI, F.

2005 L'Épipaléolithique indifférencié: caractères techno-typologiques d'un faciès mésolithique sans microlithes géométriques en Italie centro-méridionnale, en BRACCO, J.P. y MONTOYA, C. (eds.) Actes de la Table Ronde Internationale, Aix-en-Provence, 159-166.

\section{MARTZLUFF, M.}

2009 L'Azilien Pyréréen entre Garonne et Èbre: Un état de la question, en Els Pirineus i les àrees circumdants durant el Tardiglacial. Mutacions i filiacions tecnoculturals, evolució paleoambiental (16000-10000 BP). XIV Col-loqui internacional d'arqueologia de Puigcerdà. Homenatge al Professor George Laplace. Institut d'estudis ceretans, 375-422.

MARTZLUFF, M., MARTíNEZ-MORENO, J., GUILAINE, J., MORA, R. y CASANOVA, J.

2012 Transformaciones culturales y cambios climáticos en los Pirineos catalanes entre el Tardiglaciar y Holoceno antiguo: Aziliense y Sauveterriense en Balma de la Margineda y Balma Guilanyà. Cuaternario y Geomorfología, 26, 3-4, 61-78.

MAZO, C.

2006 Análisis de las huellas de uso de la serie lítica retocada del nivel IV de Mendandia, en ALDAY, A. El campamento prehistórico de Mendandia: ocupaciones mesolíticas y neolíticas entre el 8800 y el 6400 B.P. Memorias Arqueología en Castilla y León. Junta de Castilla y León, 293-327.

MAZO, C. y MONTES, L.

1992 La transición Epipaleolítico-Neolítico antiguo en el abrigo de El Pontet (Maella, Zaragoza), en UTRILLA, P. (coord.). Aragón, litoral mediterráneo. Intercambios culturales durante la Prehistoria. Encuentro de homenaje a Juan Maluquer de Motes (Zaragoza, 1990). Institución Fernando el Católico, Zaragoza.

MONTES, L.

2001/02 El abrigo epipaleolítico de Peña 14 (Biel, Zaragoza). Excavaciones 1999-2000. Saldvie, 2, 291-306.

2004 El abrigo de Legunova en Biel: Campaña de 2003. Salduie, 4, 395-406.

MONTES, L. y ALDAY, A.

2012 Enredados en la malla neolítica de la cuenca del río Ebro. Redes, continuidades y cambios. Rubricatum. Revista del Museu de Gavà, 5. Congrés Internacional Xarxes al Neolític, 51-60.

MONTES, L., UTRILLA, P. y MAZO, C.

2006 El Epipaleolítico macrolítico en Aragón en el contexto del valle del Ebro y la Cataluña costera, en ALDAY, A. (coord.) El Mesolítico de muescas y denticulados en la cuenca del Ebro y el litoral mediterráneo peninsular. Diputación Foral de Álava ed. Vol. 11. Vitoria-Gasteiz, 193-219. 
MONTES, L., DOMINGO, R., CUCHÍ, J.A., ALCOLEA, M. y SOLA, C.

2015 Completando el mapa de la Cuenca del Ebro: el Mesolítico del IX milenio cal BP de Espantalobos (Huesca, España). Munibe, 66, 119-133.

MONTES, L., DOMINGO, R., GONZÁLEZ-SAMPÉRIZ, P., SEBASTIÁN, M., ARANBARRI, J., CASTAÑOS, P., GARCÍA-SIMÓN, L.M., ALCOLEA, M. y LABORDA, R.

2016 Landscape, resources and people during Mesolithic and Neolithic times in NE Iberia: the Arba de Biel Basin. Quaternary International 403, 133-150.

MORALES, J.I., VERGÈS, J.M., FONTANALS, M., OLLÉ, A., ALLUE, E. y ANGELUCCI, D. E.

2013 Procesos técnicos y culturales durante el Holoceno inicial en el noroeste de la Península Ibérica. Los niveles $\mathrm{B}$ y $\mathrm{Bb}$ de La Cativera (El Catllar, Tarragona). Trabajos de Prehistoria, 70, 1, 54-75.

MUÑOZ, M. y BERGANZA, E.

1997 El yacimiento de la cueva de Urratxa III (Orozko, Bizkaia). Universidad de Deusto, Bilbao.

PERLES, C.

1990 Les industries lithiques taillées de Franchthi (Argolide, Grèce). Fascicle, 5. Tome II. Les industries du Mésolithique et du Néolithique Initial. Inidiana University Press.

PERRIN, T., MARCHAND, G., ALLARD, P., BINDER, D., COLLINA, C., GARCÍA PUCHOL, O. y VALDEYRON, N

2009 Le second Mésolithique d'Europe occidentale: origines et gradient chronologique. Annales de la Fondation Fyssen 24, 160-169.

RODANÉS, J. M. y. PICAZO, J. V. (coord.)

2013 El campamento mesolítico del Cabezo de la Cruz. La Muela: Zaragoza. Universidad de Zaragoza.

RODANÉS, J. M., TILO, M. A. y RAMÓN, N

1996 El abrigo de Els Secans (Mazaleón, Teruel). Campañas de excavación de 1986 y 1987. Al-Qannis, 3. Alcañiz.

RODRÍGUEZ, A.

1993 L'Analyse fonctionnelle de l'industrie du gisement épipaléolithique/mésolithique du Roc del Migdia (Catalogne, Espagne). Resultats preliminaires. Préhistoire Européenne, 4, 63-84.

RODRÍGUEZ, A. e YLL, R.

1991 Materias primas y cadenas operativas en el yacimiento epipaleolítico de El Roc del Migdia (Barcelona), en MORA, R., TERRADAS, X., PARPAL, A. y PLANA F. Tecnología y Cadenas Operativas Líticas. Treballs d'Arqueologia, I, 73-82.

SOLER, N., FULLOLA, J. Mª., SACCHI, D. y LANGLAIS, M.

2009 El Magdalenià clàssic entre Llenguadoc occidental $\mathrm{i}$ Catalunya (14500-11000 BP), en Els Pirineus i les àrees circumdants durant el Tardiglacial. Mutacions i filia- cions tecnoculturals, evolució paleoambiental (1600010000 BP). XIV Col-loqui internacional d'arqueologia de Puigcerdà. Homenatge al Professor George Laplace. Institut d'estudis ceretans, 317-348.

SOTO, A.

2014 Producción y gestión de la industria lítica de Atxoste (Álava): Una aproximación a las sociedades Epipaleolíticas y Mesolíticas del alto Ebro. Tesis doctoral. Universidad del País Vasco- Euskal Herriko Unibertsitatea.

2015 Los sistemas de producción lítica en el Epimagdaleniense y el Sauveterroide del alto Ebro: El caso del abrigo de Atxoste (Vírgala, Álava). Munibe, 93-118.

SOTO, A., ALDAY, A., MONTES, L., UTRILLA, P., PERALES, U. y DOMINGO, R.

2015 Epipaleolithic assemblages on the Ebro Basin (Spain). The difficult identification of cultural entities. Quaternary International 364, 144-152.

TARRIÑO, A.

2004 Procedencia de los sílex de la industria lítica del yacimiento de Kanpanoste (Vírgala, Álava), en CAVA, A. La ocupación prehistórica de Kanpanoste en el contexto de los cazadores-recolectores del Mesolítico. Memoria de Yacimientos Alaveses, 9, 45-60.

2006 Fuentes de aprovisionamiento de los sílex del yacimiento arqueológico de Mendandia (Sáseta, Condado de Treviño), en ALDAY, A. El campamento prehistórico de Mendandia: ocupaciones mesolíticas y neolíticas entre el 8800 y el 6400 B.P. Memorias Arqueología en Castilla y León. (Junta de Castilla y León), 473-494.

\section{TERRADAS, $X$}

1995 Las estrategias de gestión de los recursos líticos del Prepirineo catalán en el XI ${ }^{\circ}$ milenio BP: el asentamiento prehistórico de la Font del Ros (Berga, Barcelona). BeIlaterra, Universitat Autònoma de Barcelona.

UTRILLA, P.

1982/83 El yacimiento de la Cueva de Abauntz (Arraiz, Navarra). Trabajos de Arqueología de Navarra, 3, 203-345.

1995 El valle del Ebro durante el Tardiglaciar y comienzos del Holoceno. Las relaciones con el Magdaleniense cantábrico, en MOURE, A. y GONZÁLEZ SÁINZ (eds) El final del Paleolítico cantábrico, Universidad de Cantabria, 281-311.

UTRILLA, P., BERDEJO, A. y OBÓN, A.

2012 El Esplugón: un gran abrigo mesolítico en el valle del Guarga (Huesca), en MúÑIZ, J.R. Ad Orientem. Del final del Paleolítico en el norte de España a las primeras civilizaciones del Oriente próximo. Universidad de Oviedo. Ménsula Ediciones, 235-254.

UTRILLA, P., BERDEJO, A., OBÓN, A., LABORDA, R., DOMINGO, R. y ALCOLEA, M.

2016 El abrigo de El Esplugón (Billobas-Sabiñánigo, Huesca). Un ejemplo de transición Mesolítico-Neolítico en el Prepirineo central. Del neolític a l'edat del bronze en el Mediterrani occidental. Estudis en homenatge a Bernat Martí Oliver. Serie de Trabajos Varios, Servicio de Investigaciones Prehistóricas, 119, Valencia, 75-96. 
UTRILLA, P. y MAZO, C.

2014 La Peña de Las Forcas (Graus, Huesca). Un asentamiento estratégico en la confluencia del Ésera y el Isábena. Monografías Arqueológicas, 46. Universidad de Zaragoza.

UTRILLA, P., MONTES, L., MAZO, C., MARTíNEZ BEA, M. y DOMINGO, $R$.

2009 El Mesolítico geométrico en Aragón, en UTRILLA, P. y MONTES, L. (eds.). El Mesolítico Geométrico en la Península Ibérica. Monografías Arqueológicas. Prehistoria. Universidad de Zaragoza. Zaragoza, 131-190.

UTRILLA, P., MONTES, L., MAZO, C., ALDAY, A., RODANÉS, J. R., BLASCO, M. F., DOMINGO, R. y BEA, M.

2010 El Paleolítico superior en la cuenca del Ebro a principios del siglo XXI. Revisión y novedades, en MANGADO, X. El paleolítico superior peninsular. Novedades del siglo XXI. Barcelona, 23-61.

UTRILLA, P. y RODANÉS, J. M.

2004 Un asentamiento Epipaleolítico en el Valle del río Martín. El abrigo de Los Baños (Ariño, Teruel., Monografías Arqueológicas, 39. Universidad de Zaragoza.

VAQUERO, M.

2004 Els darrers caçadors-recol.lectors de la conca de Barberà: El jaciment del Molí del Salt (Vimbodí). Excavacions 1999-2003. Museu Arxiu de Montblanc i Comarca. Montblanc.

2006 El Mesolítico de facies macrolítica en el centro y sur de Cataluña, en ALDAY, A. El Mesolítico de muescas y denticulados en la cuenca del Ebro y el litoral mediterráneo peninsular. Memoria de Yacimientos Alaveses, $11,137-160$

VAQUERO, M. y GARCÍA-ARGÜELLES, P.

2009 Algunas reflexiones sobre la ausencia de Mesolítico geométrico en Cataluña, en UTRILLA, P. y MONTES, L. El mesolítico geométrico en la Península Ibérica. Monografías Arqueológicas 44, 191-204. 